\title{
Eclipsing Binaries in the Open Cluster Ruprecht 147. II. Epic 219568666
}

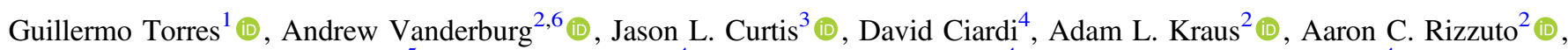 \\ Michael J. Ireland $^{5}$ (D), Michael B. Lund ${ }^{4}$ (D), Jessie L. Christiansen ${ }^{4}$ (D), and Charles A. Beichman ${ }^{4}$ \\ ${ }^{1}$ Center for Astrophysics, Harvard \& Smithsonian, 60 Garden Street, Cambridge, MA 02138, USA; gtorres@ cfa.harvard.edu \\ ${ }^{2}$ Department of Astronomy, The University of Texas at Austin, Austin, TX 78712, USA \\ ${ }^{3}$ Department of Astronomy, Columbia University, New York, NY 10027, USA \\ ${ }^{4}$ Caltech/IPAC-NASA Exoplanet Science Institute, Pasadena, CA 91125, USA \\ ${ }^{5}$ Research School of Astronomy and Astrophysics, Australian National University, Canberra, ACT 2611, Australia \\ Received 2019 October 1; revised 2019 November 4; accepted 2019 November 4; published 2019 December 16
}

\begin{abstract}
We report our spectroscopic monitoring of the detached, grazing, and slightly eccentric 12 day double-lined eclipsing binary EPIC 219568666 in the old nearby open cluster Ruprecht 147 . This is the second eclipsing system to be analyzed in this cluster, following our earlier study of EPIC 219394517. Our analysis of the radial velocities combined with the light curve from the $K 2$ mission yields absolute masses and radii for EPIC 219568666 of $M_{1}=1.121 \pm 0.013 M_{\odot}$ and $R_{1}=1.1779 \pm 0.0070 R_{\odot}$ for the F8 primary and $M_{2}=0.7334 \pm 0.0050 M_{\odot}$ and $R_{2}=0.640 \pm 0.017 R_{\odot}$ for the faint secondary. Comparison with current stellar evolution models calculated for the known metallicity of the cluster points to a primary star that is oversized, as is often seen in active M dwarfs, but this seems rather unlikely for a star of its mass and with a low level of activity. Instead, we suspect a subtle bias in the radius ratio inferred from the photometry, despite our best efforts to avoid it, which may be related to the presence of spots on one or both stars. The radius sum for the binary, which bypasses this possible problem, indicates an age of $2.76 \pm 0.61 \mathrm{Gyr}$, which is in good agreement with a similar estimate from the binary in our earlier study.
\end{abstract}

Unified Astronomy Thesaurus concepts: Eclipsing binary stars (444); Open star clusters (1160); Stellar evolutionary models (2046); Light curves (918); Fundamental parameters of stars (555); Stellar ages (1581)

Supporting material: machine-readable tables

\section{Introduction}

Detached, double-lined eclipsing binaries are the primary source of precise empirical masses and radii for normal stars (see, e.g., Andersen 1991; Torres et al. 2010). When coupled with stellar evolution models, they can also provide accurate ages. If the binary happens to be located in a cluster, the age determination serves as an important check against more traditional estimates obtained by isochrone fitting or gyrochronology, or with other techniques of dating single-member stars such as asteroseismology.

Ruprecht 147 (NGC 6774) is a nearby, middle-aged open cluster with a slightly supersolar chemical composition (distance $\sim 300 \mathrm{pc}$, age $\sim 3 \mathrm{Gyr},[\mathrm{Fe} / \mathrm{H}]=+0.10$; Curtis et al. 2013). It is endowed with no fewer than five detached eclipsing binaries identified by Curtis (2016) that are relatively bright and amenable to detailed investigation. In an earlier study, we reported results for the first of these, EPIC 219394517 (Torres et al. 2018, Paper I), a pair of very similar early G-type stars in a 6.5 day orbit that yielded highly accurate masses and radii good to better than $0.2 \%$ and $1 \%$, respectively. These properties gave an excellent fit to current stellar evolution models and an improved age for the cluster between about 2.5 and $2.6 \mathrm{Gyr}$, depending on the model, with a formal uncertainty of about $0.3 \mathrm{Gyr}$.

The present paper reports on our follow-up observations and analysis of a second eclipsing binary system in Ruprecht 147, EPIC 219568666 (TYC 6296-2012-1, 2MASS J19165992 -1625176, Gaia DR2 4183920989590558720, $V=11.86$ ), which has the potential to add significantly to the characterization of the

\footnotetext{
${ }^{6}$ NASA Sagan Fellow.
}

cluster. By virtue of the location of Ruprecht 147 on the ecliptic, EPIC 219568666 was observed along with the other eclipsing systems by NASA's $K 2$ mission in the final months of 2015 (Campaign 7). Initial follow-up by Curtis (2016) determined it to be a 12 day period, slightly eccentric, single-lined spectroscopic binary, which we find here to be double-lined. However, unlike the previous binary we reported on, the mass ratio in this case is very different from unity, which makes the secondary very faint. Membership in the cluster was established by Curtis (2016) and confirmed more recently based on parallax and proper motion information from the Gaia mission by Cantat-Gaudin et al. (2018).

As we describe below, the seemingly straightforward analysis of the high-precision $K 2$ light curve of EPIC 219568666 turns out to present difficulties that compromise the accuracy of the results. Although this does not allow us to take full advantage of the precise individual mass and radius measurements to test models of stellar evolution, the system still yields a useful estimate of the age of the cluster. This case serves as an interesting illustration of the pernicious effects of systematic errors that can easily go unnoticed, particularly those in the photometry that can bias the determination of the stellar radii in a significant way.

The layout of the paper is as follows. Section 2 describes our reduction and treatment of the raw $K 2$ photometry of EPIC 219568666, our new high-resolution spectroscopic observations, and new imaging observations to explore the field surrounding the binary. In Section 3 we present the joint spectroscopic and light curve analysis, leading to the physical properties of the system discussed in Section 4. We compare these properties with current stellar evolution models as well as with the properties of the binary in our earlier study in 
Table 1

Detrended K2 Photometry of EPIC 219568666

\begin{tabular}{lc}
\hline \hline HJD \\
$(2,400,000+)$ & Residual Flux \\
\hline 57301.4866 & 0.99987691 \\
57301.5070 & 0.99997035 \\
57301.5275 & 1.00011420 \\
57301.5479 & 1.00008800 \\
57301.5683 & 1.00009520 \\
\hline
\end{tabular}

(This table is available in its entirety in machine-readable form.)

Section 5. While this reveals discrepancies that could be due to a bias in the radius ratio preventing us from relying on the individual radii to infer an age, we are still able to make use of the radius sum, which appears to be well determined. Section 6 then discusses multiple tests to investigate the source of the discrepancies. Closing remarks are given in Section 7.

\section{Observations}

\subsection{Photometry}

EPIC 219568666 was observed by $K 2$ during its seventh observing campaign for 81 days between 2015 October and December, with a cadence of 29.4 minutes (3654 measurements). The target fell within a large super-aperture designed to observe many members of the Ruprecht 147 cluster together. Following downlink from the spacecraft and calibration by the NASA Ames $K 2$ pipeline, we proceeded to download the pixel time series from the Ruprecht 147 super-aperture from the Mikulski Archive for Space Telescopes (MAST). ${ }^{7}$ Because EPIC 219568666 is located in a dense region of stars, we extracted the light curve by calculating the flux within a concentric set of 10 circular moving apertures, to ensure that third-light contamination from nearby stars was kept constant as the telescope's pointing drifted. We performed a first-pass correction for $K 2$ 's spacecraft systematics using the methods described by Vanderburg \& Johnson (2014) and Vanderburg et al. (2016), and we selected the circular moving aperture that yielded the highest photometric precision after the systematics correction. The aperture we selected had a radius of 3.97 pixels, or $15 ! .80$. We then refined the systematics correction by simultaneously fitting for the eclipse shapes using Mandel \& Agol (2002) transit models, $K 2$ roll systematics using splines as a function of Kepler's pointing position, and long-term variability using splines in time, as described by Vanderburg et al. (2016). The resulting light curve has a scatter of about 100 parts per million (ppm) per 30 minute exposure and contains seven primary eclipses and six secondary eclipses. We removed low-frequency variability by dividing away the bestfit spline from our simultaneous light curve solution. The data processed in this way (3654 measurements) are given in Table 1.

\subsection{Imaging}

Several fainter stars near EPIC 219568666 fall within the 15 ! 80 aperture we used to extract the photometry and can potentially affect the parameters derived from the light curve. In Figure 1 we show an image of the target in a passband

\footnotetext{
http://archive.stsci.edu/
}

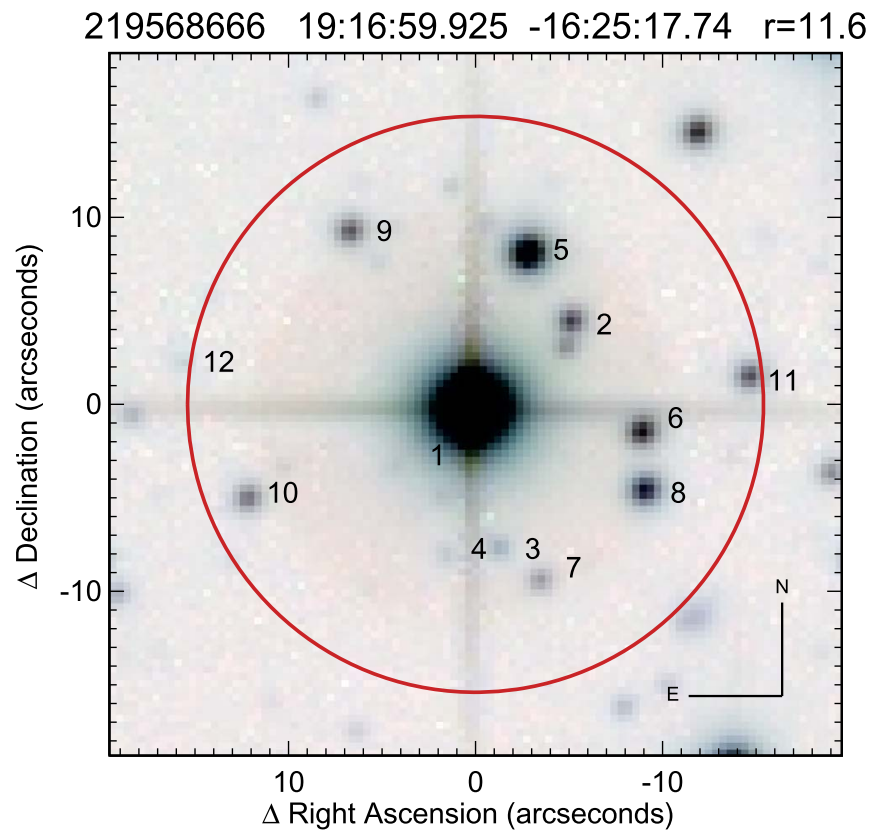

Figure 1. CFHT $r$-band image of the field of EPIC 219568666, with the 15!" 80 photometric aperture used to extract the $K 2$ photometry indicated with a circle. Nearby companions are numbered as in Table 2. Companions 1 through 4 are too faint to influence the light-curve analysis.

similar to Sloan $r$ taken in 2008 by Curtis et al. (2013) with the MegaCam instrument (Hora et al. 1994) on the CanadaFrance-Hawaii Telescope (CFHT). We measured the position relative to the target and the brightness in the CFHT gri filters ${ }^{8}$ of all numbered companions within the aperture, and we list them in Table 2. This information is used later in Section 3 to make a quantitative estimate of the flux contamination. The table includes $J$ - and $K$-band brightness measurements based on UKIRT/WFCAM imaging (Curtis 2016). We also report the properties of several of these companions that are listed in the Gaia/DR2 catalog (Gaia Collaboration et al. 2018), though none appear to be members of the cluster, based on their parallax.

In order to search for blended stellar companions to EPIC 219568666 inside the inner working angle of the CFHT imaging, we used the $10 \mathrm{~m}$ Keck II telescope with the NIRC2 facility adaptive optics (AO) imager to obtain natural guide star AO imaging and nonredundant aperture mask interferometry (NRM) in the $K^{\prime}$ filter $(\lambda=2.124 \mu \mathrm{m})$ on 2016 June 16 UT. These observations followed the standard observing strategy described by Kraus et al. (2016) and previously reported for Ruprecht 147 targets by Torres et al. (2018). For EPIC 219568666, we obtained a short sequence of six images and 12 interferograms in vertical angle mode. In both cases, calibrators were drawn from the other Ruprecht 147 members observed on the same night.

The images were analyzed following the methods described by Kraus et al. (2016). To briefly recap, the primary star pointspread function (PSF) was subtracted using both an azimuthal median profile and an independent calibrator that most closely matches the speckle pattern. Within each image, the residual fluxes as a function of position were measured in apertures of radius 40 mas, centered on each pixel, and the noise was estimated from the rms of fluxes within concentric rings around

\footnotetext{
8 http://www.cadc-ccda.hia-iha.nrc-cnrc.gc.ca/en/megapipe/docs/ filtold.html
} 
Table 2

Neighbors of EPIC 219568666

\begin{tabular}{|c|c|c|c|c|c|c|c|c|c|c|c|c|c|}
\hline$\#$ & $\begin{array}{c}\text { R.A. } \\
(\mathrm{J} 2000)\end{array}$ & $\begin{array}{c}\text { Decl. } \\
(\mathrm{J} 2000)\end{array}$ & $\begin{array}{c}\text { P.A. } \\
\text { (degree) }\end{array}$ & $\begin{array}{c}\rho \\
\left({ }^{\prime \prime}\right)\end{array}$ & $\begin{array}{c}g \\
(\mathrm{mag})\end{array}$ & $\begin{array}{c}r \\
(\mathrm{mag})\end{array}$ & $\begin{array}{c}i \\
(\mathrm{mag})\end{array}$ & $\begin{array}{l}\sigma(g r i) \\
(\mathrm{mag})\end{array}$ & $\begin{array}{c}J \\
(\mathrm{mag})\end{array}$ & $\begin{array}{c}K \\
(\mathrm{mag})\end{array}$ & $\begin{array}{l}\sigma(J K) \\
(\mathrm{mag})\end{array}$ & $\begin{array}{c}G \\
(\mathrm{mag})\end{array}$ & $\begin{array}{l}\pi_{\mathrm{DR} 2} \\
\text { (mas) }\end{array}$ \\
\hline 1 & $19: 17: 00.172$ & $-16: 25: 20.98$ & 131.8 & 4.9 & $\ldots$ & $\ldots$ & $\ldots$ & $\ldots$ & 18.38 & 17.58 & 0.05 & $\ldots$ & $\ldots$ \\
\hline 2 & $19: 16: 59.577$ & $-16: 25: 13.18$ & 312.7 & 7.0 & $\ldots$ & $\ldots$ & $\ldots$ & $\ldots$ & 17.03 & 16.62 & 0.02 & 18.59 & $0.398 \pm 0.344$ \\
\hline 3 & $19: 16: 59.832$ & $-16: 25: 25.20$ & 190.4 & 7.5 & $\ldots$ & $\cdots$ & $\ldots$ & $\ldots$ & 17.79 & 16.95 & 0.03 & 20.62 & $\ldots$ \\
\hline 4 & $19: 17: 00.023$ & $-16: 25: 25.56$ & 169.8 & 7.9 & $\ldots$ & $\ldots$ & $\ldots$ & $\ldots$ & 18.78 & 17.71 & 0.05 & $\ldots$ & $\ldots$ \\
\hline 5 & $19: 16: 59.731$ & $-16: 25: 09.50$ & 341.3 & 8.8 & 16.65 & 16.12 & 15.93 & 0.02 & 14.99 & 14.55 & 0.02 & 16.36 & $0.348 \pm 0.077$ \\
\hline 6 & $19: 16: 59.323$ & $-16: 25: 18.97$ & 262.4 & 9.1 & 17.67 & 17.26 & 17.07 & 0.02 & 16.68 & 16.25 & 0.02 & 17.98 & $0.071 \pm 0.223$ \\
\hline 7 & $19: 16: 59.686$ & $-16: 25: 26.91$ & 200.9 & 9.8 & 21.09 & 20.64 & 20.20 & 0.11 & 18.45 & 17.84 & 0.06 & 20.27 & $0.850 \pm 1.229$ \\
\hline 8 & $19: 16: 59.313$ & $-16: 25: 22.11$ & 244.1 & 10.2 & 18.43 & 17.82 & 17.54 & 0.02 & 16.23 & 15.60 & 0.02 & 17.97 & $0.401 \pm 0.184$ \\
\hline 9 & 19:17:00.361 & $-16: 25: 08.31$ & 33.4 & 11.5 & 18.72 & 18.38 & 18.29 & 0.02 & 17.54 & 17.07 & 0.03 & 18.89 & $0.021 \pm 0.311$ \\
\hline 10 & 19:17:00.724 & $-16: 25: 22.56$ & 112.4 & 12.9 & 20.06 & 19.43 & 19.17 & 0.05 & 17.77 & 17.31 & 0.04 & 19.31 & $1.280 \pm 0.455$ \\
\hline 11 & 19:16:58.939 & $-16: 25: 16.02$ & 277.2 & 14.9 & 19.60 & 18.88 & 18.63 & 0.04 & 17.25 & 16.70 & 0.02 & 18.82 & $0.293 \pm 0.317$ \\
\hline 12 & $19: 17: 00.952$ & $-16: 25: 15.29$ & 80.3 & 15.6 & 25.82 & 24.04 & 22.62 & 0.81 & 18.61 & 17.65 & 0.05 & $\ldots$ & $\ldots$ \\
\hline
\end{tabular}

Note. Coordinates derived from the astrometric solutions of the CFHT images (see Curtis et al. 2013).

(This table is available in machine-readable form.)

the primary star. Finally, the detections and detection limits were estimated from the flux-weighted sum of the detection significances in the stack of all images, and any location with a total significance greater than $6 \sigma$ was visually inspected to determine if it was a residual speckle or cosmic ray. No candidates remained after this visual inspection.

The interferograms were analyzed following the methods described by Kraus et al. (2016) and Ireland (2013). We Fouriertransformed the interferograms to extract the complex visibilities, and from those we computed the corresponding closure phases for each triplet of baselines. We calibrated the closure phases with other observations of targets nearby on the sky and in time, and we then fit the calibrated closure phases with binary source models to search for significant evidence of a companion, but did not find any. We determined the detection limits using a Monte Carlo process that randomizes the phase errors and determines the distribution of possible binary fits, indicating the $99.9 \%$ upper limit on companions in bins of projected separation.

Within the observations taken with Keck II/NIRC2 on 2016 June 16, some targets showed evidence of variable AO correction, possibly tied to variable seeing over the course of the night. In particular, some observations of EPIC 219568666 displayed an elongation of the PSF core that results when the gain in the AO system does not adapt quickly enough to changes in seeing. This resulted in little impact on the imaging limits on wide separations, but did appear to impact the sensitivity of the NRM observations.

To verify that there were no companions, we therefore made additional near-infrared high-resolution observations on 2019 June 14 UT with the PHARO instrument on the Palomar Observatory $5 \mathrm{~m}$ telescope, behind the natural guide star AO system. We used a 5-point quincunx dither pattern that is standard in this type of observation. The dither pattern step size was $5^{\prime \prime}$ and was repeated three times, with each dither offset from the previous dither by $0 . .5$.

The images were made in the narrow-band $\mathrm{Br}-\gamma$ filter $(\lambda=2.1686 \mu \mathrm{m} ; \Delta \lambda=0.0326 \mu \mathrm{m})$ with an integration time of $20 \mathrm{~s}$ and one coadd per frame for a total of $300 \mathrm{~s}$ on target. The camera was in the narrow-angle mode with a full field of view of $\sim 25^{\prime \prime}$ and a pixel scale of approximately 0 .' 025 pixel $^{-1}$. We detected no additional stellar companions to within a resolution of $\sim 0$ !" 1 FWHM (see Figure 2).

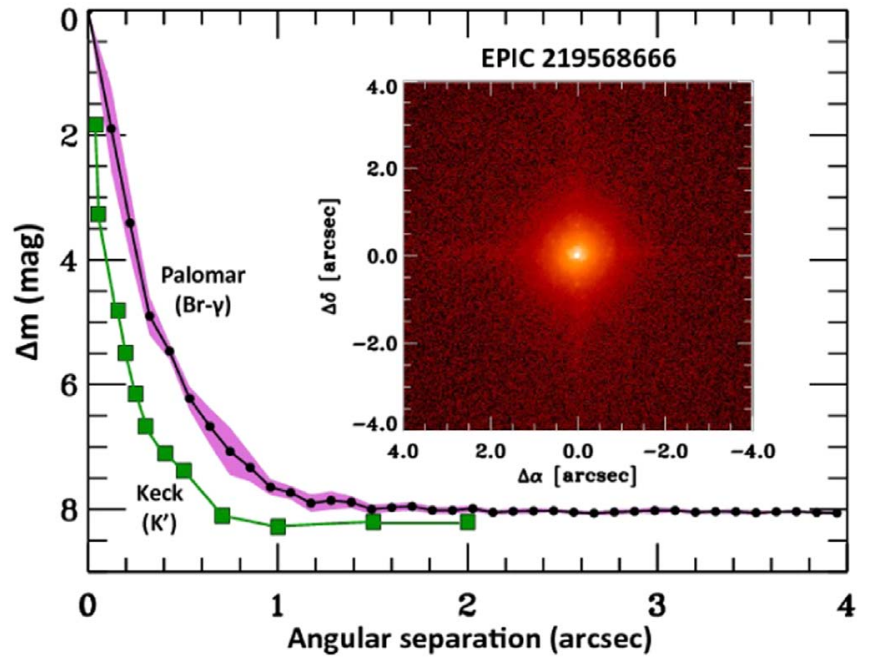

Figure 2. Companion sensitivity for our adaptive optics imaging of EPIC 219568666. The black points represent the $5 \sigma$ limits from the Palomar $\mathrm{Br}-\gamma$ observations and are separated in steps of one FWHM $\left.(\sim 0)^{\prime \prime} 1\right)$; the purple shaded area represents the azimuthal dispersion $(1 \sigma)$ of the contrast determinations (see text). The inset image of the target shows no additional companions within $4^{\prime \prime}$. The green squares represent the limits from the NIRC2 Keck observations at $K^{\prime}$, which also revealed no companions.

The sensitivity of the final combined Palomar AO image was determined by injecting simulated sources azimuthally around the target every $45^{\circ}$ at separations of integer multiples of the central source's FWHM (Furlan et al. 2017). The brightness of each injected source was scaled until standard aperture photometry detected it with $5 \sigma$ significance. The resulting brightness of the injected sources relative to the target set the contrast limits at that injection location. The final $5 \sigma$ limit at each separation was determined from the average of all of the determined limits at that separation, and the uncertainty on the limit was set by the rms dispersion of the azimuthal slices at a given radial distance.

The sensitivity curves from the Keck II/NIRC2 and Palomar/PHARO observations are displayed in Figure 2 along with an inset image zoomed in on the target, showing no other companion stars. 


\subsection{Spectroscopy}

Spectroscopic monitoring of EPIC 219568666 was carried out between 2016 May and 2018 June with the Tillinghast Reflector Echelle Spectrograph (TRES; Szentgyorgyi \& Fürész 2007; Fûrész 2008) on the $1.5 \mathrm{~m}$ Tillinghast reflector at the Fred L. Whipple Observatory on Mount Hopkins (Arizona, USA). We gathered a total of 25 spectra at a resolving power of $R \approx 44,000$ covering the wavelength region $3800-9100 \AA$ in 51 orders. For the order centered at $\sim 5187 \AA$ containing the $\mathrm{Mg}$ I b triplet, the signal-to-noise ratios range from 17 to 42 per resolution element of $6.8 \mathrm{~km} \mathrm{~s}^{-1}$.

Radial velocities were measured with TODCOR, a twodimensional cross-correlation technique introduced by Zucker \& Mazeh (1994). A template matching each component was selected from a precomputed library of synthetic spectra based on model atmospheres by R. L. Kurucz and a line list tuned to better match the spectra of real stars (see Nordström et al. 1994; Latham et al. 2002). These templates cover a limited wavelength region of $\sim 300 \AA$ centered at $5187 \AA$.

For the primary star, we estimated the effective temperature $\left(T_{\text {eff }}\right)$ and projected rotational velocity $(v \sin i)$ by running grids of one-dimensional cross-correlations of the observed spectra against synthetic spectra, following Torres et al. (2002). The grids were run over a broad range in those parameters $\left(4500-7500 \mathrm{~K}, 0-30 \mathrm{~km} \mathrm{~s}^{-1}\right)$ at fixed values of the surface gravity $(\log g)$ and metallicity $([\mathrm{Fe} / \mathrm{H}])$, ignoring the presence of the secondary star because it is very faint (only about $4 \%$ of the brightness of the primary; see below). We then chose the combination giving the highest value of the cross-correlation coefficient averaged over all 25 spectra, weighted by the strength of each exposure. We repeated this for $\log g$ values of 4.0 and 4.5 , bracketing our best estimate reported later, and $[\mathrm{Fe} / \mathrm{H}]$ values of 0.0 and +0.5 on either side of the known cluster abundance. By interpolation, we obtained estimates of $T_{\text {eff }}=6140 \mathrm{~K}$ and $v \sin i=6 \mathrm{~km} \mathrm{~s}^{-1}$, with estimated uncertainties of $100 \mathrm{~K}$ and $2 \mathrm{~km} \mathrm{~s}^{-1}$, respectively. These uncertainties are based on the scatter from the individual spectra, conservatively increased to account for possible systematic errors. The temperature corresponds approximately to a spectral type of F8. For the velocity determinations, we adopted primary template parameters of $6250 \mathrm{~K}$ and $6 \mathrm{~km} \mathrm{~s}^{-1}$, the nearest in our grid, along with $\log g=4.5$ and $[\mathrm{Fe} / \mathrm{H}]=0.0$.

While the presence of the secondary does not affect the above determinations, its faintness does prevent us from obtaining direct estimates of its temperature and $v \sin i$ from our spectra. We therefore adopted a value of $T_{\text {eff }}=4500 \mathrm{~K}$ appropriate for its mass (typical of a mid-K star) and $v \sin i=4 \mathrm{~km} \mathrm{~s}^{-1}$. The surface gravity and metallicity for the secondary template were taken to be the same as for the primary.

The heliocentric radial velocities measured with TODCOR are presented in Table 3 along with their uncertainties. We also determined the average flux ratio at the mean wavelength of our observations $\left(5187 \AA\right.$ ), which is $\ell_{2} / \ell_{1}=0.044 \pm 0.003$. A graphical representation of the velocities is shown in Figure 3, along with our final model described below. The orbit is clearly eccentric. We note that two observations near phase 0.46 (the first and third in Table 3) are safely outside of eclipse and are thus not affected by the Rossiter-McLaughlin effect.

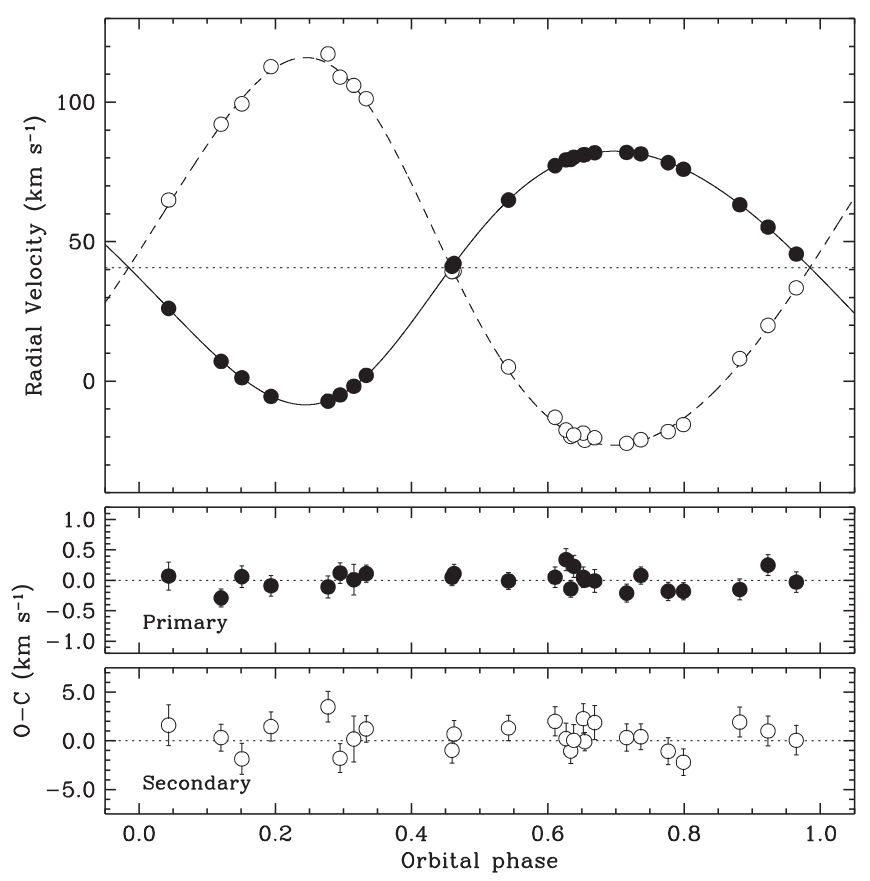

Figure 3. Radial-velocity measurements for EPIC 219568666 with our adopted model. Primary and secondary measurements are represented with filled and open circles, respectively. The dotted line marks the center-of-mass velocity of the system. Error bars are too small to be visible. They are seen in the lower panels, which display the residuals. Phases are counted from the reference time of primary eclipse.

Table 3

Heliocentric Radial-velocity Measurements of EPIC 219568666

\begin{tabular}{lrcrcc}
\hline \hline $\begin{array}{l}\text { HJD } \\
(2,400,000+)\end{array}$ & $\begin{array}{c}\mathrm{RV}_{1} \\
\left(\mathrm{~km} \mathrm{~s}^{-1}\right)\end{array}$ & $\begin{array}{c}\sigma_{1} \\
\left(\mathrm{~km} \mathrm{~s}^{-1}\right)\end{array}$ & $\begin{array}{c}\mathrm{RV}_{2} \\
\left(\mathrm{~km} \mathrm{~s}^{-1}\right)\end{array}$ & $\begin{array}{c}\sigma_{2} \\
\left(\mathrm{~km} \mathrm{~s}^{-1}\right)\end{array}$ & $\begin{array}{c}\text { Orbital } \\
\text { Phase }\end{array}$ \\
\hline 57528.9254 & 42.17 & 0.15 & 39.43 & 1.39 & 0.4623 \\
57550.9028 & -4.96 & 0.17 & 108.99 & 1.48 & 0.2951 \\
57552.8726 & 41.13 & 0.14 & 39.26 & 1.31 & 0.4594 \\
57553.8662 & 64.90 & 0.14 & 5.10 & 1.29 & 0.5422 \\
57554.9623 & 79.50 & 0.14 & -19.80 & 1.28 & 0.6336 \\
57555.9456 & 81.95 & 0.15 & -22.28 & 1.39 & 0.7157 \\
57556.9458 & 75.93 & 0.14 & -15.55 & 1.36 & 0.7991 \\
57557.9371 & 63.21 & 0.17 & 8.08 & 1.53 & 0.8817 \\
57558.9318 & 45.53 & 0.17 & 33.42 & 1.52 & 0.9647 \\
57566.8695 & 79.32 & 0.18 & -17.51 & 1.58 & 0.6266 \\
57583.8587 & 26.07 & 0.23 & 64.89 & 2.08 & 0.0434 \\
57584.7819 & 7.08 & 0.15 & 92.09 & 1.38 & 0.1204 \\
57700.5724 & 78.30 & 0.15 & -18.06 & 1.39 & 0.7766 \\
57705.5734 & -5.50 & 0.17 & 112.75 & 1.51 & 0.1937 \\
57706.5771 & -7.17 & 0.18 & 117.29 & 1.57 & 0.2774 \\
57710.5737 & 77.23 & 0.17 & -12.98 & 1.50 & 0.6107 \\
57854.9921 & 81.17 & 0.10 & -21.17 & 0.93 & 0.6542 \\
57878.9487 & 81.09 & 0.17 & -18.60 & 1.51 & 0.6521 \\
57908.9128 & 1.21 & 0.18 & 99.40 & 1.58 & 0.1509 \\
57910.8838 & -1.82 & 0.25 & 106.00 & 2.35 & 0.3153 \\
57939.9190 & 81.46 & 0.14 & -20.99 & 1.32 & 0.7366 \\
58034.6680 & 80.25 & 0.18 & -19.28 & 1.62 & 0.6381 \\
58274.8628 & 81.86 & 0.19 & -20.29 & 1.74 & 0.6688 \\
58277.9134 & 55.22 & 0.17 & 19.99 & 1.54 & 0.9232 \\
58294.8240 & 2.07 & 0.14 & 101.25 & 1.35 & 0.3335 \\
\hline & & & & &
\end{tabular}

Note. Orbital phases are counted from the reference time of primary eclipse. Final velocity uncertainties result from scaling the values listed for the primary and secondary by the near-unity factors $f_{1}$ and $f_{2}$, respectively, from our global analysis described in Section 3.

(This table is available in machine-readable form.) 


\section{Analysis}

The light-curve analysis of EPIC 219568666 was carried out using the eb code of Irwin et al. (2011), which is based on the Nelson-Davis-Etzel binary model (Etzel 1981; Popper \& Etzel 1981) implemented in the popular EBOP program and its descendants. Further details may be found in our earlier work (Torres et al. 2018). The main adjustable parameters are the orbital period $(P)$ and reference epoch of primary eclipse $\left(T_{0}\right.$, which is strictly the time of inferior conjunction in this code), the central surface brightness ratio in the Kepler bandpass $\left(J \equiv J_{2} / J_{1}\right)$, the sum of the relative radii normalized by the semimajor axis $\left(r_{1}+r_{2}\right)$ and their ratio $\left(k \equiv r_{2} / r_{1}\right)$, the cosine of the inclination angle $(\cos i)$, and the eccentricity parameters $e \cos \omega$ and $e \sin \omega$, with $e$ being the eccentricity and $\omega$ the longitude of periastron of the primary. We adopted a quadratic limb-darkening law for this work, with coefficients $u_{1}$ and $u_{2}$ for each star.

Our detrending procedure is designed to eliminate the obvious modulation in the light curve that is due to spots, as well as any long-term trends (deemed to be of instrumental origin), and at the same time it removes other out-of-eclipse variability including tidal distortions (ellipsoidal variability) and reflection, which for this long-period and well-detached system are very small in any case. Therefore, our modeling was run with the calculation of ellipsoidal variability and reflection disabled, and we restricted the analysis to data within 0.02 in phase from the center of each eclipse (about 2.5 times the total duration of the eclipses). We accounted for the finite time of integration of the $K 2$ long-cadence observations by oversampling the model light curve and then integrating over the 29.4 minute duration of each cadence prior to the comparison with the observations (see Gilliland et al. 2010; Kipping 2010).

Our method of solution used the emcee $e^{9}$ code of ForemanMackey et al. (2013), which is a Python implementation of the affine-invariant Markov Chain Monte Carlo (MCMC) ensemble sampler proposed by Goodman \& Weare (2010). We used 100 walkers with chain lengths of 10,000 each, after discarding the burn-in. Uniform (noninformative) or log-uniform priors over suitable ranges were adopted for all parameters (see below), and convergence of the chains was checked visually, requiring also a Gelman-Rubin statistic of 1.05 or smaller for each parameter (Gelman \& Rubin 1992).

Flux contamination from neighboring stars was accounted for by including a third light parameter in our model $\left(\ell_{3}\right)$, defined such that $\ell_{1}+\ell_{2}+\ell_{3}=1$, in which the values of $\ell_{1}$ and $\ell_{2}$ for this normalization correspond to the light at first quadrature (phase 0.2235 in this system). An estimate of $\ell_{3}$ was obtained from the brightness measurements in Table 2 of all companions within the photometric aperture, using the magnitudes in the CFHT $r$ band, which is close to the Kepler bandpass. To guard against possible errors arising from the slight bandpass difference, we conservatively inflated the companion magnitude uncertainties to be no less than $0.2 \mathrm{mag}$. In this way we obtained $\ell_{3}=0.026 \pm 0.006$, which we used as a Gaussian prior in our MCMC analysis. All companions near the edge of the circular aperture are very faint, so the result is insensitive to the treatment of partial pixels for those stars.

In eccentric orbits such as that of EPIC 219568666, it is usually the case that $e \cos \omega$ is tightly constrained by the light curve (from the location of the secondary eclipse), while $e \sin \omega$

\footnotetext{
https://github.com/dfm/emcee
}

is not. The reverse is true of the radial-velocity curves, making it beneficial to combine the two types of observations into a joint solution. We took this approach, solving for three additional adjustable parameters: the center-of-mass velocity $(\gamma)$ and the velocity semiamplitudes of the primary and secondary $\left(K_{1}\right.$ and $\left.K_{2}\right)$. We handled the relative weighting between the photometry and the primary and secondary velocities by including additional adjustable multiplicative parameters $\left(f_{K 2}, f_{1}\right.$, and $f_{2}$, respectively) to rescale the observational errors. These scale factors were solved for selfconsistently and simultaneously with the other orbital quantities (see Gregory 2005). The initial error assumed for the photometric measurements is $200 \mathrm{ppm}$, and the initial errors for the velocities are those listed in Table 3.

For completeness, we chose to account for light travel time across the binary, which can contribute to the displacement of the secondary eclipse from phase 0.5 , although the effect is negligible in this case: a delay of $26 \mathrm{~s}$, more than three orders of magnitude smaller than the measured displacement corresponding to about $54,800 \mathrm{~s}$. Strictly speaking, then, our $T_{0}$ is referred to the barycenter of the binary.

As shown below, the eclipses of EPIC 219568666 are partial and quite shallow ( $\sim 11 \%$ and $\sim 4 \%)$. With the expectation that the radius ratio $k$ would be poorly constrained, as it often is in such cases, we chose to take advantage of our spectroscopic measurement of the average light ratio to help constrain $k$ indirectly, given that the two quantities are strongly correlated $\left(\ell_{2} / \ell_{1} \propto k^{2}\right)$. Our measured value of $\ell_{2} / \ell_{1}=0.044 \pm 0.003$ at a mean wavelength of $5187 \AA$ was transformed to the Kepler band by using synthetic spectra based on PHOENIX models taken from the grid of Husser et al. (2013). For this we adopted temperatures of 6100 and $4500 \mathrm{~K}$, close to those of the components, and a preliminary estimate of the radius ratio $(k=0.60)$, with which we are able to reproduce the measured light ratio at $5187 \AA$. The result, $\ell_{2} / \ell_{1}=0.084 \pm 0.005$, was then applied as a prior in our analysis.

Initial solutions revealed that the second-order limb-darkening coefficients $u_{2}$ were poorly constrained for both stars. This is to be expected given the grazing orientation of the system (see below). Therefore, for the remainder of this work, they were held fixed at the theoretical values tabulated by Claret \& Bloemen (2011), interpolated according to the adopted temperatures, the metallicity, and our final surface gravities reported below. The values of $u_{2}$ adopted for the Kepler band are 0.295 and 0.075 for the primary and secondary, respectively, and are based on ATLAS model atmospheres and the least-squares procedure favored by those authors for calculating the coefficients.

We report the results of our analysis in Table 4, where the values given correspond to the mode of the posterior distributions. Posterior distributions of the derived quantities listed in the bottom section of the table were constructed directly from the MCMC chains of the adjustable parameters involved. Our adopted model along with the $K 2$ observations can be seen in Figure 4, together with an illustration of the fairly grazing configuration of the system. The residuals, with an overall scatter of about $120 \mathrm{ppm}$, appear slightly larger in the secondary eclipse. This may be due to spottedness, which would not be unexpected for a mid-K star such as the secondary.

To account for the possibility that the increased secondary residuals (or the fact that we held the second-order limbdarkening coefficients fixed) may be causing our parameter 
Table 4

Results from Our Combined MCMC Analysis for EPIC 219568666

\begin{tabular}{|c|c|c|}
\hline Parameter & Value & Prior \\
\hline$P$ (days) & $11.991313_{-0.000013}^{+0.000013}$ & {$[11,13]$} \\
\hline$T_{0}(\mathrm{HJD}-2,400,000)$ & $57727.23362_{-0.00042}^{+0.00042}$ & {$[57725,57729]$} \\
\hline$J$ & $0.2707_{-0.0051}^{+0.0051}$ & {$[0.02,1.0]$} \\
\hline$r_{1}+r_{2}$ & $0.06715_{-0.00045}^{+0.00045}$ & {$[0.01,0.20]$} \\
\hline$k$ & $0.544_{-0.016}^{+0.016}$ & {$[0.1,1.0]$} \\
\hline $\cos i$ & $0.04128_{-0.00064}^{+0.00064}$ & {$[0,1]$} \\
\hline$e \cos \omega$ & $-0.083091_{-0.000013}^{+0.000013}$ & {$[-1,1]$} \\
\hline$e \sin \omega$ & $-0.0747_{-0.0010}^{+0.0010}$ & {$[-1,1]$} \\
\hline$\ell_{3}$ & $0.0261_{-0.0061}^{+0.0060}$ & {$[0.0,0.3]$} \\
\hline$\gamma\left(\mathrm{km} \mathrm{s}^{-1}\right)$ & $+40.732_{-0.033}^{+0.033}$ & {$[30,50]$} \\
\hline$K_{1}\left(\mathrm{~km} \mathrm{~s}^{-1}\right)$ & $45.440_{-0.044}^{+0.044}$ & {$[20,80]$} \\
\hline$K_{2}\left(\mathrm{~km} \mathrm{~s}^{-1}\right)$ & $69.46_{-0.37}^{+0.37}$ & {$[20,80]$} \\
\hline $\ln f_{K 2}$ & $-0.479_{-0.044}^{+0.047}$ & {$[-5,0.9]$} \\
\hline $\ln f_{1}$ & $+0.09_{-0.14}^{+0.17}$ & {$[-5,5]$} \\
\hline $\ln f_{2}$ & $-0.04_{-0.14}^{+0.15}$ & {$[-5,5]$} \\
\hline Primary $u_{1}$ & $0.291_{-0.064}^{+0.064}$ & {$[0,1]$} \\
\hline Secondary $u_{1}$ & $0.455_{-0.064}^{+0.063}$ & {$[0,1]$} \\
\hline \multicolumn{3}{|c|}{ Derived quantities } \\
\hline$r_{1}$ & $0.04349_{-0.00022}^{+0.00021}$ & $\cdots$ \\
\hline$r_{2}$ & $0.02364_{-0.00059}^{+0.00061}$ & $\cdots$ \\
\hline$i$ (degree) & $87.634_{-0.036}^{+0.037}$ & $\cdots$ \\
\hline$\ell_{2} / \ell_{1}$ & $0.0781_{-0.0037}^{+0.0039}$ & $\ldots$ \\
\hline$e$ & $0.11176_{-0.00069}^{+0.00069}$ & $\ldots$ \\
\hline$\omega$ (degree) & $221.98_{-0.41}^{+0.38}$ & $\cdots$ \\
\hline$f_{K 2}$ & $0.620_{-0.027}^{+0.029}$ & $\ldots$ \\
\hline$f_{1}$ & $1.09_{-0.14}^{+0.20}$ & $\ldots$ \\
\hline$f_{2}$ & $0.95_{-0.12}^{+0.16}$ & $\cdots$ \\
\hline Primary duration (days) & $0.20800_{-0.00047}^{+0.00047}$ & $\cdots$ \\
\hline Secondary duration (days) & $0.19582_{-0.00062}^{+0.00062}$ & $\cdots$ \\
\hline Primary impact param. & $1.012_{-0.019}^{+0.020}$ & $\cdots$ \\
\hline Secondary impact param. & $0.872_{-0.017}^{+0.017}$ & $\cdots$ \\
\hline
\end{tabular}

Note. The values listed correspond to the mode of the respective posterior distributions, and the uncertainties represent the $68.3 \%$ credible intervals that include a contribution from extra photometric noise possibly caused by stellar activity (see text). All priors are uniform over the specified ranges, except those for $f_{K 2}, f_{1}$, and $f_{2}$, which are $\log$-uniform. Eclipse durations are counted from first to last contact.

uncertainties to be underestimated, we performed a residual permutation exercise as follows. We shifted the residuals from our adopted model by an arbitrary number of time indices, we then added them back into the model curve at each time of observation (with wrap-around), and we carried out a new MCMC analysis on this synthetic data set. We repeated this 50 times. The residual permutation was done separately for the in-eclipse and out-of-eclipse regions (see Hartman et al. 2018). For each new MCMC analysis, we simultaneously perturbed the theoretical quadratic limb-darkening coefficients by adding Gaussian noise with a standard deviation of 0.10 . The scatter (standard deviation) of the resulting distributions for each parameter was added in quadrature to the internal errors from our original MCMC analysis to arrive at the final uncertainties reported in Table 4. The simulation errors are larger than the internal errors for $P, T_{0}$, and the linear limb-darkening coefficients.
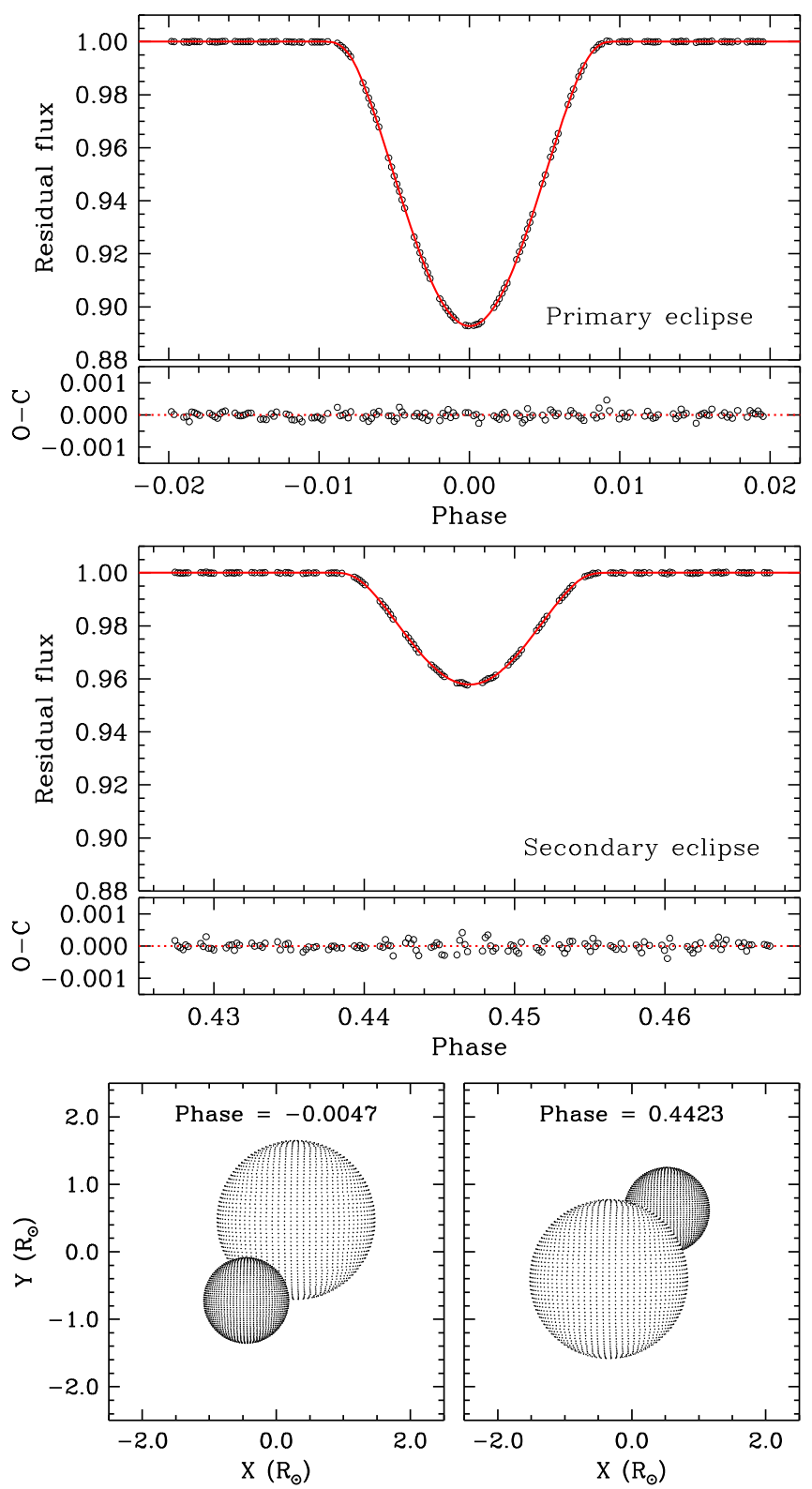

Figure 4. Top two panels: $K 2$ observations of EPIC 219568666 and our adopted model. Residuals are shown at the bottom for each eclipse. Bottom: illustration of the grazing configuration of the system near each of the eclipses, as projected on the sky (star sizes drawn to scale).

We note, finally, that our analysis reveals strong correlations among several of the fitted elements, which is not unexpected for a system of this configuration. The strongest correlations are between $k$ and $r_{1}+r_{2}$ (correlation coefficient +0.921$)$, between $k$ and $\cos i(+0.938)$, between $r_{1}+r_{2}$ and $\cos i(+0.992)$, and between $P$ and $T_{0}(+0.997)$.

\section{Absolute Dimensions}

The physical properties of EPIC 219568666 derived from our MCMC analysis are collected in Table 5. The relative uncertainties for the masses are $\sim 1 \%$ or smaller. The radii are formally good to $0.6 \%$ and $2.5 \%$ for the primary and secondary, although we argue below that systematic errors may be larger. 
Table 5

Physical Properties of EPIC 219568666

\begin{tabular}{|c|c|c|}
\hline Parameter & Primary & Secondary \\
\hline$\overline{M\left(\mathcal{M}_{\odot}^{\mathrm{N}}\right)}$ & $1.121_{-0.013}^{+0.013}$ & $0.7334_{-0.0049}^{+0.0050}$ \\
\hline$R\left(\mathcal{R}_{\odot}^{\mathrm{N}}\right)$ & $1.1779_{-0.0070}^{+0.0070}$ & $0.640_{-0.016}^{+0.017}$ \\
\hline$q \equiv M_{2} / M_{1}$ & \multirow{2}{*}{\multicolumn{2}{|c|}{$\begin{array}{c}0.6542_{-0.0036}^{+0.0036} \\
27.086_{-0.089}^{+0.089}\end{array}$}} \\
\hline$a\left(\mathcal{R}_{\odot}^{\mathrm{N}}\right)$ & & \\
\hline $\log g(\operatorname{dex})$ & $4.3457_{-0.0048}^{+0.0049}$ & $4.690_{-0.022}^{+0.022}$ \\
\hline$T_{\text {eff }}(\mathrm{K})$ & $6140 \pm 100$ & $4500 \pm 100$ \\
\hline$L\left(L_{\odot}\right)$ & $1.77_{-0.11}^{+0.12}$ & $0.152_{-0.015}^{+0.016}$ \\
\hline$M_{\mathrm{bol}}(\mathrm{mag})$ & $4.109_{-0.072}^{+0.072}$ & $6.78_{-0.11}^{+0.11}$ \\
\hline$B C_{V}$ (mag) & $-0.027 \pm 0.100$ & $-0.624 \pm 0.100$ \\
\hline$M_{V}(\mathrm{mag})$ & $4.14_{-0.12}^{+0.12}$ & $7.40_{-0.15}^{+0.15}$ \\
\hline$v_{\mathrm{sync}} \sin i\left(\mathrm{~km} \mathrm{~s}^{-1}\right)^{\mathrm{a}}$ & $4.966_{-0.029}^{+0.029}$ & $2.699_{-0.067}^{+0.070}$ \\
\hline$v \sin i\left(\mathrm{~km} \mathrm{~s}^{-1}\right)^{\mathrm{b}}$ & $6 \pm 2$ & 4 (adopted) \\
\hline$E(B-V)(\mathrm{mag})$ & \multicolumn{2}{|c|}{$0.126 \pm 0.023$} \\
\hline$A_{V}(\mathrm{mag})$ & \multicolumn{2}{|c|}{$0.391 \pm 0.071$} \\
\hline Dist. modulus (mag) & \multicolumn{2}{|c|}{$7.39_{-0.15}^{+0.15}$} \\
\hline Distance $(\mathrm{pc})$ & \multicolumn{2}{|c|}{$300_{-19}^{+21}$} \\
\hline$\pi$ (mas) & \multicolumn{2}{|c|}{$3.33_{-0.22}^{+0.23}$} \\
\hline$\pi_{\text {Gaia } / \mathrm{DR} 2(\mathrm{mas})^{\mathrm{c}}}$ & \multicolumn{2}{|c|}{$3.277 \pm 0.052$} \\
\hline
\end{tabular}

Notes. The masses, radii, and semimajor axis $a$ are expressed in units of the nominal solar mass and radius $\left(\mathcal{M}_{\odot}^{\mathrm{N}}, \mathcal{R}_{\odot}^{\mathrm{N}}\right)$, as recommended by 2015 IAU Resolution B3 (see Prša et al. 2016), and the adopted solar temperature is 5772 K (2015 IAU Resolution B2). Bolometric corrections are from the work of Flower (1996), with conservative uncertainties of $0.1 \mathrm{mag}$, and the bolometric magnitude adopted for the Sun appropriate for this $B C_{V}$ scale is $M_{\text {bol }}^{\odot}=4.732$ (see Torres 2010). See text for the source of the reddening. For the apparent visual magnitude of EPIC 219568666 out of eclipse, we used $V=11.859 \pm 0.050$ (Zacharias et al. 2013).

${ }^{a}$ Synchronous projected rotational velocity assuming spin-orbit alignment.

${ }^{\mathrm{b}}$ Measured projected rotational velocity for the primary.

${ }^{\mathrm{c}}$ A global zero-point correction of +0.029 mas has been added to the parallax (Lindegren et al. 2018a), and 0.021 mas is added in quadrature to the internal error (see Lindegren et al. 2018b).

As described earlier, the primary effective temperature was determined directly from our spectra, while the secondary value is adopted. These temperatures along with broadband photometry from the literature allow us to obtain an estimate of the interstellar reddening along the line of sight. We proceeded as follows. With the values in Table 5, the luminosity-weighted spectroscopic mean temperature of the system is $\left\langle T_{\text {eff }}\right\rangle_{\mathrm{sp}}=5877 \pm 84 \mathrm{~K}$. Color indices from published photometry along with color/temperature calibrations allow a determination of a mean photometric temperature, $\left\langle T_{\text {eff }}\right\rangle_{\mathrm{ph}}$, which depends on the overall reddening, $E(B-V)$. We used photometry in the Johnson, Tycho-2, 2MASS, and Sloan systems (Høg et al. 2000; Skrutskie et al. 2006; Zacharias et al. 2013; Henden et al. 2015) to construct 13 nonindependent color indices, and we derived a temperature for each one using the calibrations by Casagrande et al. (2010) and Huang et al. (2015). Following Torres et al. (2018), we adjusted the temperatures from the Casagrande et al. (2010) calibrations by $-130 \mathrm{~K}$ to remove a systematic difference compared to those of Huang et al. (2015). The results were then averaged. The metallicity terms in these calibrations were calculated using $[\mathrm{Fe} / \mathrm{H}]=+0.10 \pm 0.04$ (Curtis et al. 2013), and the reddening corrections appropriate for each color index were made as prescribed by Cardelli et al. (1989). We determined the optimal reddening $E(B-V)$ by varying it until $\left\langle T_{\text {eff }}\right\rangle_{\mathrm{ph}}=\left\langle T_{\text {eff }}\right\rangle_{\mathrm{sp}}$. In this way we obtained $E(B-V)=0.126 \pm 0.023 \quad \mathrm{mag}$,
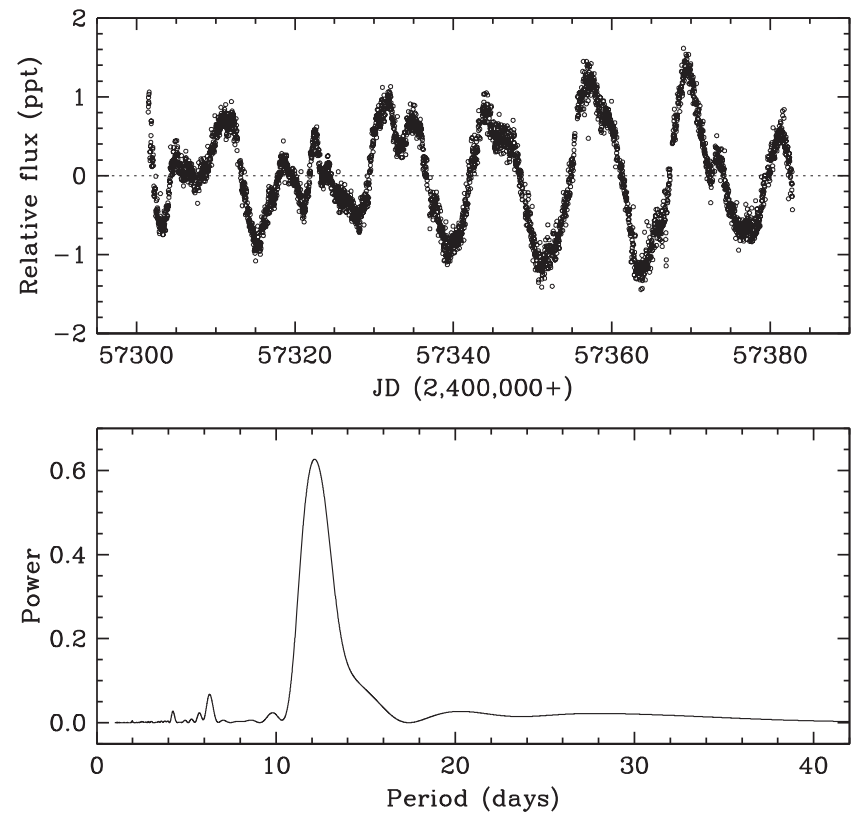

Figure 5. Top: rotational modulation in the light curve of EPIC 219568666 (relative flux in parts per thousand, ppt). Eclipses have been removed for clarity, along with a long-term instrumental drift. Bottom: Lomb-Scargle periodogram of the observations displaying a dominant peak at $P_{\text {rot }}=12.2_{-0.9}^{+1.1}$ days.

corresponding to $A_{V}=0.391 \pm 0.071 \mathrm{mag}$ for a ratio of total to selective extinction of $R_{V}=3.1$. This is in good agreement with the value of $E(B-V)=0.112 \pm 0.029$ mag derived in our earlier study of the Ruprecht 147 eclipsing system EPIC 219394517, and also with independent estimates for the cluster by Bragaglia et al. (2018) and Olivares et al. (2019).

The distance to EPIC 219568666 was estimated from the luminosities, bolometric corrections from Flower (1996; see also Torres 2010), our extinction estimate, and an adopted visual magnitude out of eclipse of $V=11.859 \pm 0.050$ (Zacharias et al. 2013). The result, $d=300_{-20}^{+21} \mathrm{pc}$, corresponds to a parallax in good accord with the entry in the Gaia/DR2 catalog (Gaia Collaboration et al. 2018, see Table 5). Finally, given the $\sim 3$ Gyr age of the cluster, the nonzero eccentricity of the binary $(e \approx 0.112)$ is consistent with the expectation from tidal theory, which predicts an orbital circularization timescale (formally 630 Gyr; e.g., Hilditch 2001) that is longer than the age of the universe.

\subsection{Rotation and Activity}

The light curve of EPIC 219568666 presents obvious variations out of eclipse with a peak-to-peak amplitude of about 2 mmag. We attribute this to rotational modulation by spots, although it is unclear which star is responsible. While it may well be the brighter primary, the secondary is likely to be spotted as well, as suggested by the somewhat larger photometric residuals during secondary eclipse. In that case, the dilution effect would imply an intrinsic amplitude from spots on the secondary of about $2.5 \%$ in the Kepler band.

The observations after removal of the eclipses and a longterm drift are presented in Figure 5, along with the corresponding Lomb-Scargle periodogram. The peak location at $P_{\mathrm{rot}}=12.2_{-0.9}^{+1.1}$ days (uncertainties estimated from the half width at half maximum) is consistent with the orbital period, suggesting synchronous rotation. Our spectroscopically 

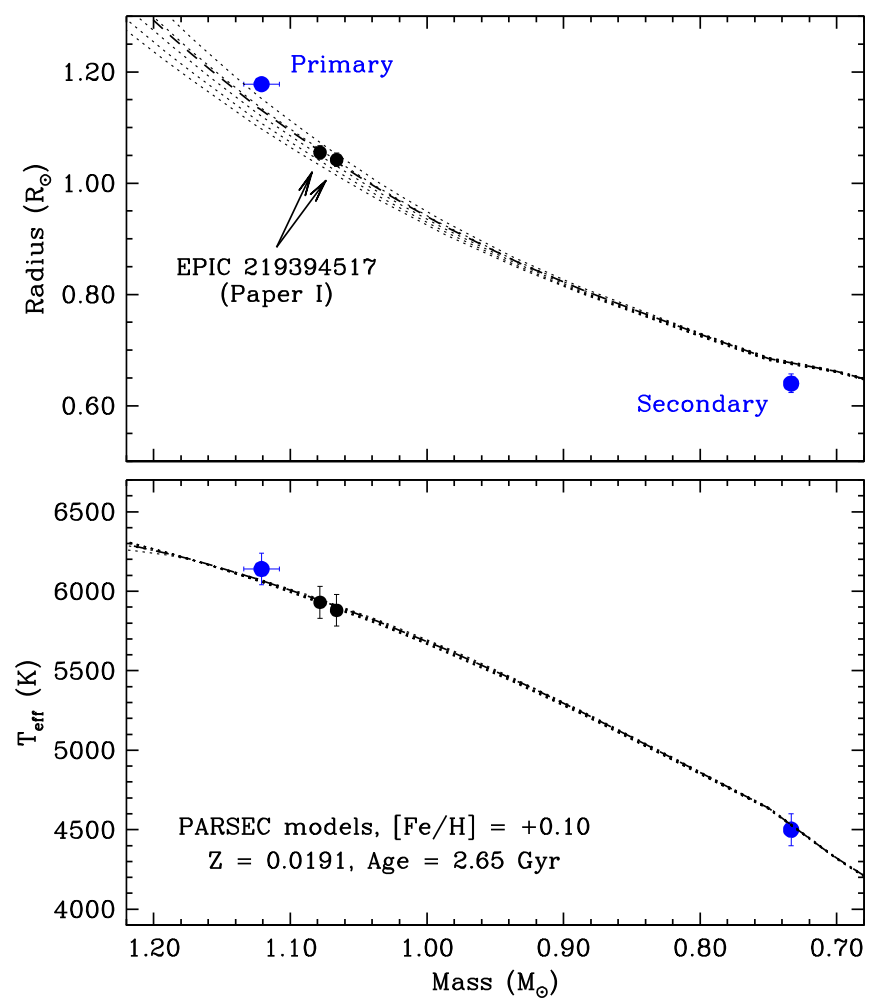

Figure 6. Comparison of the measured masses, radii, and effective temperatures of EPIC 219568666 (blue points) against stellar evolution models from the PARSEC series (Chen et al. 2014). We show also the results for the EPIC 219394517 system (smaller black points) from our earlier study (Torres et al. 2018). The adopted metallicity is that of the Ruprecht 147 cluster, $[\mathrm{Fe} / \mathrm{H}]=+0.10$. Dotted lines correspond to model isochrones from 2 to $3 \mathrm{Gyr}$ in equally spaced logarithmic intervals, and the heavier dashed line represents the best-fit age of 2.65 Gyr from our earlier work on EPIC 219394517. The error bars shown are the nominal values from Table 4 , which do not reflect correlations discussed in the text.

measured projected rotational velocity of the primary star $\left(v \sin i=6 \pm 2 \mathrm{~km} \mathrm{~s}^{-1}\right.$ ) agrees with the predicted value of $v_{\text {sync }} \sin i$ shown in Table 5. Both of these indications are consistent with the expected synchronization timescale of \%300 Myr (e.g., Hilditch 2001), given the $~ 3$ Gyr age of the parent Ruprecht 147 cluster.

Aside from the small photometric modulation described above, the activity level of the binary appears to be low, as we see no indicators of such activity in our spectra, nor does the object appear to have been detected in X-rays.

\section{Comparison with Theory}

Our precise determinations of the masses and radii of EPIC 219568666, and the increased leverage afforded by a mass ratio significantly different from unity, offer an opportunity for a valuable comparison with current stellar evolution models. They also permit an independent estimate of the age of the Ruprecht 147 cluster to supplement the one from our previous study of EPIC 219394517 (Torres et al. 2018). Figure 6 displays the measured radius and temperature of the components as a function of their measured mass, together with model isochrones from the PARSEC series (Chen et al. 2014) computed for the cluster metallicity of $[\mathrm{Fe} / \mathrm{H}]=+0.10$. For reference, we include the results for EPIC 219394517 from Paper I. The model isochrones are the same as shown in Figure 7 of that work and

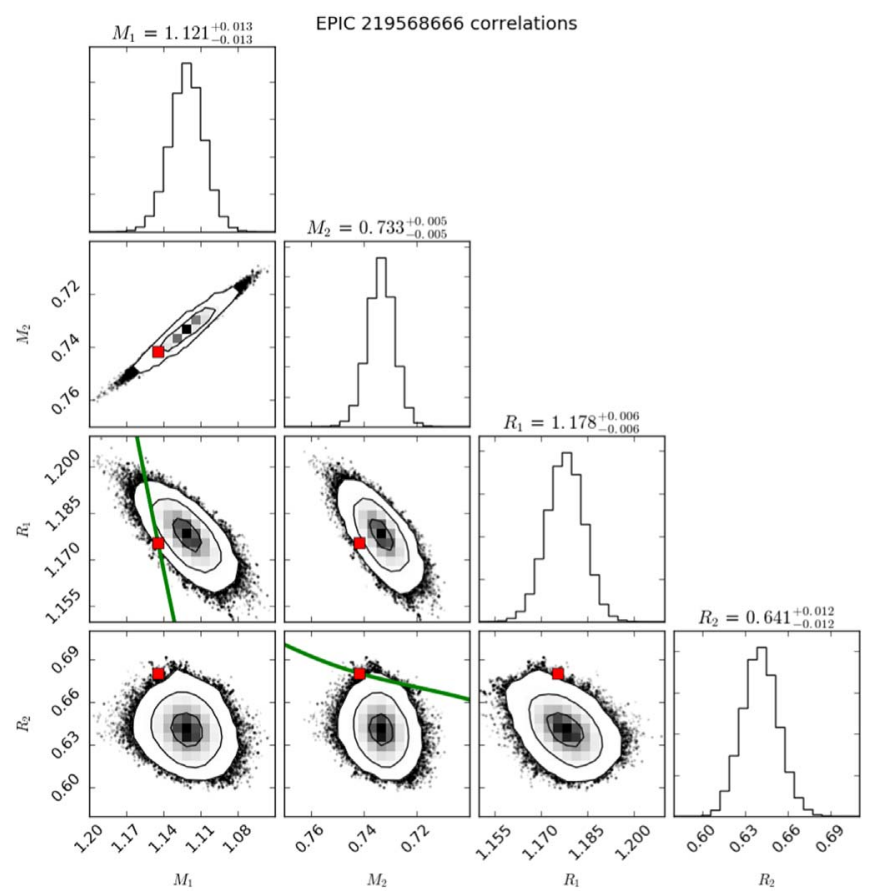

Figure 7. "Corner" plot (Foreman-Mackey 2016) (https://github.com/dfm/ corner.py) based on the results of our MCMC analysis showing the correlations among the derived masses and radii of EPIC 219568666. The contours correspond to the $1 \sigma, 2 \sigma$, and $3 \sigma$ confidence levels. The green diagonal lines in the $\left[M_{1}, R_{1}\right]$ and $\left[M_{2}, R_{2}\right]$ panels are segments of the reference isochrone from Figure 6. The red squares mark the points on the isochrone with the smallest normalized difference in four-dimensional space relative to the measured mass and radius values in Table 4 (see text).

span ages between 2 and 3 Gyr. The heavy dashed line represents the $2.65 \mathrm{Gyr}$ isochrone that provided the best fit to EPIC 219394517 in our earlier study.

While the isochrones match the effective temperature of the primary of EPIC 219568666 to within its uncertainty (the secondary $T_{\text {eff }}$ is adopted from models), the radius of the primary appears $\sim 4 \%$ larger than expected for its mass $(6.7 \sigma)$ if we take the best-fitting model from EPIC 219394517 as the reference. Furthermore, the radius of the secondary is about $6 \%$ smaller than the models predict $(2.2 \sigma)$, assuming the mass is accurate. These differences are somewhat surprising, particularly for the primary, given the high precision of the observations, the relatively straightforward analysis, and the expectation of fewer complications from the longer orbital period compared to our previous study of the more active 6.5 day system EPIC 219394517. Repeating the comparison with the MIST models (Choi et al. 2016) gives essentially the same result.

A casual look at Figure 6 may give the impression that relatively small shifts in the locations of the primary and secondary in just the right directions would bring satisfactory agreement with the reference model from EPIC 219394517. For instance, an increase in $M_{1}$ by about $2 \sigma$ together with an increase in $R_{2}$ also by about $2 \sigma$ would be sufficient to obtain a reasonably good fit. However, this ignores the strong correlations that exist among the inferred masses and radii, which restrict the shifts one may apply in each direction if they are to remain within the multidimensional confidence region mapped out by our MCMC analysis. As an example, $M_{1}$ and $M_{2}$ have a correlation coefficient of +0.981 , indicating they cannot be varied independently. 
A more quantitative assessment of how well the measurements agree with the reference isochrone that implicitly accounts for all correlations may be obtained using the chains from our emcee analysis, with a combined length of $10^{6}$ links. For each link, we determined the normalized distance in fourdimensional parameter space between the values of $\left[M_{1}, M_{2}\right.$, $R_{1}, R_{2}$ ] and the nearest pair of points on the isochrone representing the location of the primary and secondary. We normalized the separation along each axis by the standard deviation of the corresponding variable as determined from the chains. The masses and radii on the isochrone that are closest to the link giving the smallest distance are represented with square symbols in Figure 7. This figure displays a "corner" plot showing the correlations among the four quantities. The contours correspond to the $1 \sigma, 2 \sigma$, and $3 \sigma$ confidence levels, and the panels for $\left[M_{1}, R_{1}\right]$ and $\left[M_{2}, R_{2}\right]$ also show the reference isochrone from Figure 6 . The square symbols in the latter two panels deviate from the values reported in Table 4 by more than $2 \sigma$ for the primary and more than $3 \sigma$ for the secondary. Similar offsets are seen in the other panels. The overall discrepancy with the model is at about the $3 \sigma$ level, which we consider significant.

If we assume for a moment that the masses of both stars are unbiased, the positive deviation in radius for the primary star may be speculated to be due to stellar activity, which is believed to be the underlying cause of the phenomenon of "radius inflation" in convective stars (see, e.g., Mullan \& MacDonald 2001; Chabrier et al. 2007; Feiden \& Chaboyer 2013, 2014; Torres 2013; Somers \& Pinsonneault 2015). We note, though, that the activity level of the primary seems relatively low, based on the photometric amplitude of the rotational modulation (provided it comes from this star and not the secondary) and on the lack of spectroscopic activity indicators ( $\mathrm{H} \alpha$ or $\mathrm{Ca}$ II $\mathrm{H}$ and $\mathrm{K}$ emission) or X-ray emission. Furthermore, radius inflation is most often seen in $\mathrm{K}$ and $\mathrm{M}$ dwarfs, and much more rarely among higher-mass convective stars near a solar mass, though a few examples do exist (e.g., the secondary components of CV Boo, FL Lyr, V1061 Cyg, and V636 Cen, with masses of $0.97,0.96,0.93$ and $0.85 M_{\odot}$, respectively; see Torres et al. 2008, 2006; Clausen et al. 2009; Hełminiak et al. 2019). None are as massive as the F8 primary of EPIC 219568666, however, and all rotate more rapidly.

On the other hand, we can think of no plausible physical explanation for the small radius of the secondary at its nominal mass, although the deviation is admittedly less significant than for the primary. Instead, we postulate that the discrepancies for both stars are more likely to be due to systematic errors stemming from our analysis or from the observations themselves. In the next section, we discuss possible sources of these errors and the tests we carried out to investigate them.

We note here that light-curve solutions for systems with shallow eclipses such as EPIC 219568666 can often lead to a biased measure of the radius ratio (especially, but not necessarily, when the components are similar, which is not the case here) because of degeneracies among several orbital elements (see, e.g., Andersen 1991; Torres et al. 2010; Kraus et al. 2015). Such degeneracies are present in our own analysis, as pointed out at the end of Section 3. On the other hand, the sum of the radii is typically more robust (e.g., Andersen et al. 1983). Interestingly, we estimate that an increase from the value of $k \approx 0.54$ we determine to about 0.60 would yield very good agreement with the models shown in Figure 6. This may

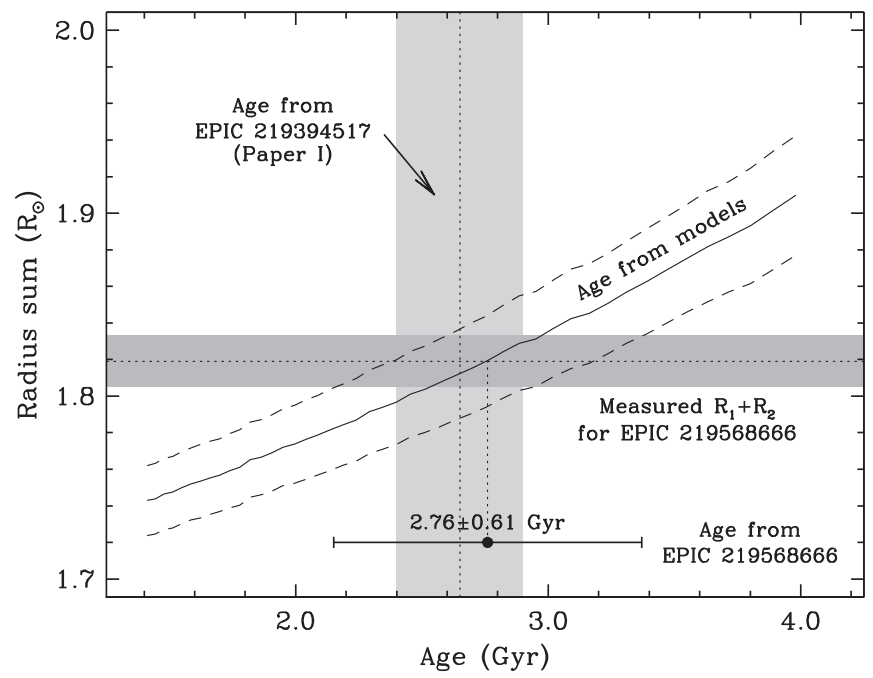

Figure 8. Age determination for EPIC 219568666 based on the radius sum (dot and error bars, $2.76 \pm 0.61 \mathrm{Gyr}$ ). The horizontal shaded area represents the measured value of $R_{1}+R_{2}$ and its uncertainty. The age from the PARSEC models along with the $1 \sigma$ uncertainty resulting from the errors in the measured masses are shown with the solid and dashed lines. The age determined from our earlier study of EPIC 219394517 (Torres et al. 2018) is shown for reference by the vertical shaded area.

be taken as circumstantial evidence of a possible bias in the radius ratio, although the situation is likely more complex given the correlations between $k$ and other elements.

If we set aside for now the individual radii and rely only on the radius sum $\left(R_{1}+R_{2}=1.819 \pm 0.014 R_{\odot}\right)$, which we expect to be more accurate, it is still possible to estimate an age for the system using the same PARSEC models as above. This is illustrated in Figure 8, in which the solid curve gives the age predicted from theory (for the adopted cluster metallicity of $[\mathrm{Fe} / \mathrm{H}]=+0.10)$ as a function of the radius sum computed at the measured masses for EPIC 219568666. The corresponding $1 \sigma$ error interval that comes from the uncertainty in the measured masses is shown with dashed lines. The intersection of this curve with the measured radius sum for EPIC 219568666 (horizontal shaded area) leads to an age estimate of $2.76 \pm 0.61 \mathrm{Gyr}$ (dot and error bars at the bottom). Although the uncertainty is more than twice that of the estimate for EPIC 219394517 from Paper I $(2.65 \pm 0.25 \mathrm{Gyr}$ at fixed metallicity), the determinations are consistent within the smallest of their errors. In both cases we used the same models. Together the two binaries therefore support an age for the cluster slightly under 3 Gyr.

\section{Discussion}

In this section, we examine a number of possible explanations for the unexpected disagreement illustrated in Figure 6 between models and the mass and radius measurements for EPIC 219568666, which appears statistically significant, as discussed above. Barring an unusual instance of (activityrelated?) radius inflation for a star as massive as the primary, or severely underestimated mass or radius uncertainties that we think are unlikely, we proceed on the assumption that there may be a subtle bias somewhere in our analysis that is causing the primary to appear to be too large and the secondary too small compared to predictions, or perhaps the primary mass to be too small. We address potential errors in the spectroscopy, errors in the priors for the light ratio and third light that we 
applied in our MCMC analysis, the possibility of biases in our detrending procedures for the $K 2$ photometry, and the effect of the cadence of the photometric observations.

\subsection{Mass Errors}

Earlier we proposed that an underestimate of the radius ratio is one plausible explanation for the measured slope in the mass-radius diagram being too steep (Figure 6). In principle, a bias in the mass ratio may contribute as well. This could result from a mismatch between the templates used in our TODCOR analysis (involving mainly the $T_{\text {eff }}$ parameter, which affects the velocities the most) and the spectra of the real components. While the primary temperature is well determined directly from our spectra, the secondary temperature $(4500 \mathrm{~K})$ was adopted from models because that star is too faint for an independent determination. We repeated the radial-velocity measurements for a range of temperatures for the secondary template between 4000 and $5000 \mathrm{~K}$ in steps of $250 \mathrm{~K}$, but we found that the residuals from a spectroscopic orbital solution were worse; the masses changed by less than $1 \%$ compared to the adopted values, and the mass ratio changed by even less. We conclude from this that the masses appear to be robust and are therefore not likely to be the main source of the discrepancy, though they may still have some influence. This then shifts the focus to the radii, which depend more critically on the photometry.

\subsection{Alternate Solutions}

A separate MCMC analysis without the radial velocities gave essentially the same results for both the radius sum and radius ratio, again suggesting that the problem may lie in the photometry. Splitting the photometric data set in two produced similar parameters in each half, from which we conclude any bias is not time-dependent.

\subsection{Priors}

Our adopted solution in Table 4 used Gaussian priors for both the light ratio and third light, each based on empirical constraints. To investigate the effect of these priors, we repeated the analysis without them, as well as using one prior but not the other. All three solutions resulted in values of $k$ and $r_{1}+r_{2}$ (and also of $\ell_{2} / \ell_{1}$ and $\ell_{3}$ ) that were very close to those obtained when applying both priors together, suggesting little or no tension between the photometry and the external constraints. Nevertheless, we examined each of the priors more closely.

\subsubsection{Spectroscopic Light Ratio}

The prior on the light ratio can potentially have the strongest effect on the inferred value of the radius ratio because of the direct correlation between them $\left(\ell_{2} / \ell_{1} \propto k^{2}\right)$. For example, grids of MCMC solutions imposing Gaussian light-ratio priors ranging from 0.030 to 0.150 (straddling our adopted value of $0.084 \pm 0.005)$ and the same prior on $\ell_{3}$ as in our original analysis show indeed that the derived $k$ values change significantly from 0.50 to 0.73 (a $46 \%$ change), while the values of $r_{1}+r_{2}$ change in tandem but less, from about 0.066 to $0.071(8 \%)$, supporting the notion that the radius sum is more robust. Removing the $\ell_{3}$ prior in this exercise causes the radius sum to change in the opposite direction, from 0.071 to 0.066 . However, in both cases, the quality of the solution as measured by the $f_{K 2}$ scale factor for the photometric errors degrades considerably toward the upper end of the $\ell_{2} / \ell_{1}$ range we explored.

Our adopted light-ratio prior in the Kepler band is extrapolated from our measured value at $\sim 5187 \AA$, as described in Section 3, and as such it is subject to error. The extrapolation used synthetic spectra by Husser et al. (2013) appropriate for each component in order to calculate the wavelength dependence of the flux ratio. The normalization was performed using $k=0.60$, which is the value of the radius ratio we find is needed in order to reproduce the measured $\ell_{2} / \ell_{1}$ value at $5187 \AA(0.044 \pm 0.003)$. Interestingly, this value of $k$ (which is independent of our MCMC analysis) is also the one that seems to provide a match between the PARSEC evolutionary models and the individual masses and radii of EPIC 219568666, assuming $r_{1}+r_{2}$ is accurate. As noted above, however, our MCMC analysis returns a lower $k$ value close to 0.54 , not 0.60 .

As a check on our extrapolation, we repeated the light-ratio determination with TODCOR in other echelle orders available in our spectra sampling the entire Kepler bandpass. Because our standard template library (Nordström et al. 1994; Latham et al. 2002) only spans about $300 \AA$ around the region of the Mg I b triplet, we used synthetic spectra based on PHOENIX models from the same Husser et al. (2013) library as above that cover the entire optical range. We note that these synthetic spectra were not used for our original radial-velocity determinations because at high resolution they do not match real stars as well as the templates from our own library, which are based on a line list tuned for that purpose. Nevertheless, the Husser et al. (2013) library suffices for a determination of the light ratio, which does not require high resolution.

We measured $\ell_{2} / \ell_{1}$ in 31 spectral orders between 4300 and $8900 \AA$ that have high enough flux and are sufficiently free from telluric lines. The results are displayed in Figure 9 as points with error bars. Also shown is the predicted flux ratio from the synthetic spectra for two different values of the radius ratio. The top curve is for $k=0.60$, the value required to reproduce the measured light ratio at $5187 \AA$, and the bottom curve is for $k=0.54$, which is our result from the light-curve analysis. The open squares on the top $k=0.60$ curve mark the predicted values at $5187 \AA$ (matching the spectroscopic $0.044 \pm 0.003$ measurement by construction) and at the Kepler band $(0.084 \pm 0.005)$. They were computed by integrating the theoretical flux ratio over the corresponding response functions (assumed to have a top-hat shape for the $\mathrm{MgIb}$ order). The Kepler band prediction for the $k=0.54$ curve is marked with an open circle. For reference, the shaded area represents the response function for Kepler with arbitrary normalization.

Our order-by-order light-ratio measurements over the Kepler band clearly agree best with the upper $(k=0.60)$ curve in Figure 9 and thus support the accuracy of the $\ell_{2} / \ell_{1}$ prior used in our analysis, which was based on that value of the radius ratio. At the same time, this purely spectroscopic approach conflicts with the result for $k$ returned by our MCMC analysis, suggesting the latter value is underestimated. We note that this conclusion is independent of the stellar evolution models.

\subsubsection{Third Light}

Our Gaussian prior for $\ell_{3}(0.026 \pm 0.006)$ relies on brightness measurements for all known stars within the photometric aperture, based on a seeing-limited image from the CFHT (Figure 1 and Section 3). While our higher-resolution AO 


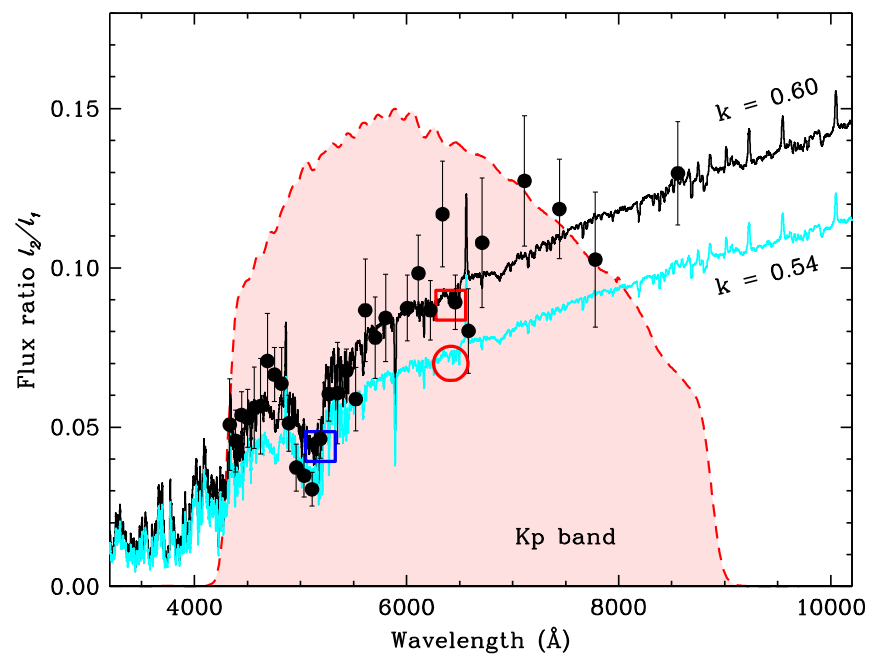

Figure 9. Spectroscopic flux ratio measurements (points with error bars) compared with calculations based on synthetic spectra for solar metallicity from Husser et al. (2013), for component temperatures of 6100 and $4500 \mathrm{~K}$ and $\log g=4.5$. Two model predictions are shown. One (black) was normalized using a radius ratio of $k=0.60$, a value such that the curve integrated over the spectral order containing the $\mathrm{Mg} \mathrm{I} b$ triplet returns a value (blue square) matching the measured light ratio at $5187 \AA$. The other model (cyan) was normalized using a radius ratio of $k=0.54$, which is the value resulting from our MCMC analysis (Table 4). The predictions of both models for the Kepler band are represented by the red square and circle, respectively. The Kepler response function is also shown.

imaging in Section 2.2 revealed no additional companions outside of $0 . " 1$, it is still possible we are missing flux that is due to even closer companions. To explore this possibility and its effect on the analysis, we ran a grid of MCMC solutions in which we varied the $\ell_{3}$ prior between 0.03 and 0.12 , maintaining our light-ratio prior as in our original analysis. We found only a very small increase in $k(0.539-0.544)$ coupled with a reduction in $r_{1}+r_{2}(0.0670-0.0654)$, which together result in an even smaller value for the secondary radius than indicated in Table 5. Furthermore, if the missing third light were due to a physically associated single star, a value as high as $\ell_{3}=0.12$ would imply a brightness for that star relative to the primary of $\ell_{3} / \ell_{1} \approx 0.13$ in the Kepler band, which is even brighter than the secondary $\operatorname{star}\left(\ell_{2} / \ell_{1}=0.084\right)$. There is no sign of such a tertiary in our spectra, nor do we see any trend in the radial-velocity residuals from our spectroscopic orbit that might indicate an outer companion bound to the binary.

\subsection{Limb Darkening}

For the present analysis we adopted the quadratic limbdarkening law for both stars, which is commonly used when dealing with space-based photometry. We allowed the firstorder coefficients to vary and held the second-order coefficients fixed from theory because they are poorly constrained by the data given that the eclipses are grazing. The first-order coefficient we obtain for the primary, $0.291 \pm 0.064$, is quite consistent with the theoretical value of 0.343 from Claret \& Bloemen (2011), while the one for the secondary star, $0.455 \pm 0.064$, is lower than expected (0.674). In principle, the use of a different or possibly more sophisticated limbdarkening prescription could affect the resulting geometric parameters to some extent, particularly the radius sum and radius ratio. Unfortunately, we are not able to test alternate formulations here because the public eb code we use restricts our choices to either the linear or quadratic laws. Nevertheless, as an experiment, we repeated the analysis with the linear law and found that neither $k$ nor $r_{1}+r_{2}$ changed significantly. The linear coefficients we derived in this case for the primary and secondary, $0.464 \pm 0.012$ and $0.428 \pm 0.034$, may be compared with the theoretical values of 0.564 and 0.730 , respectively. Using the linear law for one star and the quadratic law for the other also did not affect the geometric parameters in any meaningful way. While these experiments do not completely rule out the possibility that a different or higherorder limb-darkening formula might have a more significant impact, the fact that even reverting to the simpler linear law did not change the results makes that seem less likely. In retrospect this is not all that surprising, as the grazing configuration means that the photometry typically has very little discriminating power on the details of limb darkening.

\subsection{Detrending}

Given the above evidence that neither our spectroscopy nor our Gaussian priors on $\ell_{2} / \ell_{1}$ or $\ell_{3}$ seem to be causing a bias in our light-curve analysis, suspicion falls on the photometry itself, and in particular on the processing (detrending) to which it was subjected prior to analysis.

We therefore tested different ways of treating the photometry, focusing both on the systematics-removal stage and on the removal of low-frequency variability, to see if residual systematic errors or subtle biases from our low-frequency removal might explain the discrepancy between our best-fit radius ratio and the radii predicted by evolutionary models.

First, we tried removing low-frequency variability from the $K 2$ light curve in a different way. Instead of dividing away the best-fit spline from the light curve solution, we fit for a new spline after the removal of systematics while manually masking the eclipses. We then tweaked our systematics-removal process to see if subtle biases from our simultaneous fit to the eclipse shapes, the $K 2$ systematics, and low-frequency variability were causing a bias in the radius ratio. We tested the light curve with only a first-pass systematics correction, and we found that the best-fit radius ratio from this light curve was essentially unchanged compared to our original processing.

We also tested different light curves produced by simultaneously fitting the systematics and low-frequency variability along with more sophisticated models for the eclipses. The Mandel \& Agol (2002) code we used in our detrending was designed for modeling the light curves of transiting planets and assumes that each set of transits or eclipses has the same limbdarkening profile, and furthermore, it does not include a thirdlight contribution. In our tests, we relaxed both of these assumptions by including a third-light contribution in the eclipse model and by allowing the flexibility for the code to fit each set of eclipses with their own quadratic limb-darkening coefficients. Interestingly, none of these changes significantly affected the best-fit radius ratio $k$ or the radius sum $r_{1}+r_{2}$ derived from the light curve.

\subsection{Impact of the Radius Ratio on the Light Curve}

In order to quantify the impact that a change in the radius ratio has on the light curve itself, we compared our adopted model with another in which we forced $k$ to be approximately 0.60 . We did this by applying a tight prior on $k$ and removing the prior on $\ell_{2} / \ell_{1}$ so as to avoid conflict. All other parameters 
were solved for in the same way as before. The difference between the synthetic light curves from these two models has a maximum amplitude of about $100 \mathrm{ppm}$ during the ingress/ egress of the secondary eclipse, and less than half of that during the primary eclipse. We note that this is of the same order as the overall scatter of the $K 2$ photometry from our adopted model $(\sim 120 \mathrm{ppm})$. We speculate that this difference may be too small for our analysis to detect given the photometric noise.

\subsection{Cadence of the Photometric Observations}

Finally, we explored the possibility that the relatively long 29.4 minute sampling of the $K 2$ light curve may be negatively affecting our results, given that only a handful of observations were recorded near the points of first and last contact (see Figure 4), which are critical for defining the radii. For this we used the same $k=0.60$ solution mentioned in the previous section and oversampled the corresponding model to emulate the 1 minute short-cadence (SC) observations that $K 2$ has obtained for many targets (but not for EPIC 219568666). As a check, we also sampled it at the same 29.4 minute rate (long cadence, LC) as the real observations. We then added Gaussian noise appropriate to each cadence and subjected the two synthetic data sets to identical MCMC analyses as with the real data, integrating the model over 29.4 minutes for the LC time series but not for the SC time series. We repeated this several times, each with different noise, and found that within the uncertainties both synthetic data sets gave the same results for $k$ and $r_{1}+r_{2}$, on average, and that those results were also similar to the input values of those parameters for this experiment. We conclude that the cadence of the photometric observations has little influence in this particular case.

\subsection{Summary}

From the tests described above, we conclude that the apparent bias in the radius ratio suggested by Figure 6 and supported by Figure 9 does not seem to be caused by errors in the radial-velocity measurements, by incorrect priors on $\ell_{2} / \ell_{1}$ or $\ell_{3}$, by the subtleties of our detrending procedures, or by the finite cadence of the observations. The change in $k$ required to match the slope in the mass-radius diagram (from $\sim 0.54$ to $\sim 0.60$ ) is about $11 \%$ of the value we measure. While this change may seem significant at face value (about 3.7 times the formal uncertainty in $k$ ), the effect on the shape of the light curve is actually very small, as indicated earlier, and it may be that the data available are insufficient to discern this difference. Another possibility that cannot be ruled out has to do with the presence of spots on one or both stars, especially given the grazing configuration of the EPIC 219568666 system. Spots can cause systematic errors in the measured eclipse depths (changing the surface brightness ratio), which in turn can lead to biases in other geometric parameters such as the radius ratio, the inclination angle, and even the radius sum. An example with a much more detailed discussion of spot effects on eclipsing binary parameters may be found in the work of Irwin et al. (2011).

\section{Concluding Remarks}

EPIC 219568666 is the second eclipsing binary we have analyzed in the Ruprecht 147 cluster after EPIC 219394517 (Torres et al. 2018), based on $K 2$ photometry and follow-up spectroscopic observations. While the formal precision of our mass and radius determinations is quite high $(\sim 1 \%$ relative errors in the masses, and about $0.6 \%$ and $2.5 \%$ errors in the radii), the agreement with stellar evolution models is less satisfactory for EPIC 219568666 than it was for the system we studied earlier. The primary star seems too large for its mass (a $6.7 \sigma$ discrepancy), while the secondary is a bit too small (though only by $2.2 \sigma$ ). Activity-related radius inflation seems unlikely for the primary for the reasons indicated earlier, and there is no evidence of any strong systematic errors in the masses, which leads us to suspect a bias in the radius ratio we inferred from the $K 2$ light curve.

A weakly constrained radius ratio is not an uncommon occurrence in light-curve analyses, though it often goes unnoticed. The usual cure for this problem is the use of an external constraint on the light ratio derived, for example, from spectroscopy, as we have done here. For EPIC 219568666, this does not seem to have completely eliminated biases, despite our best attempts. Careful examination of the critical ingredients for the mass and radius determinations, as detailed in the preceding section, has given no clues as to what could be causing $k$ to be underestimated in our analysis. A remaining possibility is a bias in the geometric parameters caused by spots on one or both stars. Simulations by Morales et al. (2010) have shown that these effects appear to be maximized when the spots are concentrated at the poles. Although we have no information on the latitudinal distribution of spots in this system, it is possible that acquiring multicolor observations might help, as the different sensitivity to spots as a function of wavelength could help break degeneracies with other effects such as limb darkening, particularly in a grazing configuration such as that of the present system.

The example of EPIC 219568666 serves as a cautionary tale about the need to be aware of the potential for biases in the solution of light curves, especially regarding properties that are often poorly constrained such as the radius ratio. This can even affect photometric measurements from space-based missions, which may well be internally highly precise but could still suffer from subtle systematic errors not reflected in the formal uncertainties. Such errors could result, for example, from the complex processing that space photometry is typically subjected to prior to use, or from physical effects such as the presence of spots, as mentioned above. Independent highprecision photometric observations of EPIC 219568666 such as may be obtained in the future with NASA's Transiting Exoplanet Survey Satellite (TESS), or at other wavelengths from the ground, may shed some light on this issue.

We have shown that it is still possible to infer a useful estimate of the age of EPIC 219568666 by relying only on the sum of the radii, bypassing the use of the individual radii involving $k$. The result, $2.76 \pm 0.61 \mathrm{Gyr}$, at a metallicity fixed to that of the cluster, is consistent with the independent estimate obtained in our earlier study of EPIC 219394517 $(2.65 \pm 0.25 \mathrm{Gyr})$, if much less precise. Together these two age estimates therefore point to an age for Ruprecht 147 near 2.7 Gyr. We expect to strengthen this determination even further as studies are completed for three additional eclipsing binaries in the cluster that are underway.

The spectroscopic observations of EPIC 219568666 were gathered with the help of P. Berlind, M. Calkins, G. Esquerdo, 
and D. Latham. J. Mink is thanked for maintaining the CfA echelle database. We are also grateful to J. Irwin for implementing changes in the eb program that facilitated the present analysis, and to the anonymous referee for helpful comments and suggestions. G.T. acknowledges partial support from NASA's Astrophysics Data Analysis Program through grant 80NSSC18K0413, and to the National Science Foundation (NSF) through grant AST-1509375. J.L.C. is supported by the NSF Astronomy and Astrophysics Postdoctoral Fellowship under award AST-1602662, and by NASA under grant NNX16AE64G issued through the $K 2$ Guest Observer Program (GO 7035). This work was performed in part under contract with the California Institute of Technology (Caltech)/Jet Propulsion Laboratory (JPL) funded by NASA through the Sagan Fellowship Program executed by the NASA Exoplanet Science Institute. The research has made use of the SIMBAD and VizieR databases, operated at the CDS, Strasbourg, France, and of NASA's Astrophysics Data System Abstract Service. The research was made possible through the use of the AAVSO Photometric All-Sky Survey (APASS), funded by the Robert Martin Ayers Sciences Fund. Data products were also used from the Two Micron All Sky Survey, which is a joint project of the University of Massachusetts and the Infrared Processing and Analysis Center/California Institute of Technology, funded by NASA and the NSF. The work has also made use of data from the European Space Agency (ESA) mission Gaia (https://www. cosmos.esa.int/gaia), processed by the Gaia Data Processing and Analysis Consortium (DPAC, https://www.cosmos.esa.int/ web/gaia/dpac/consortium). Funding for the DPAC has been provided by national institutions, in particular the institutions participating in the Gaia Multilateral Agreement. The computational resources used for this research include the Smithsonian Institution's "Hydra" High Performance Cluster.

\section{ORCID iDs}

Guillermo Torres (iD https://orcid.org/0000-0002-5286-0251 Andrew Vanderburg (iD https://orcid.org/0000-00017246-5438

Jason L. Curtis (1) https://orcid.org/0000-0002-2792-134X Adam L. Kraus (D) https://orcid.org/0000-0001-9811-568X Aaron C. Rizzuto (D) https://orcid.org/0000-0001-9982-1332 Michael J. Ireland (i) https://orcid.org/0000-0002-6194-043X Michael B. Lund (1) https://orcid.org/0000-0003-2527-1598 Jessie L. Christiansen (i) https://orcid.org/0000-00028035-4778

\section{References}

Andersen, J. 1991, A\&ARv, 3, 91

Andersen, J., Clausen, J. V., Nordström, B., et al. 1983, A\&A, 121, 271

Bragaglia, A., Fu, X., Mucciarelli, A., Andreuzzi, G., \& Donati, P. 2018, A\&A, 619, A176

Cantat-Gaudin, T., Jordi, C., Vallenari, A., et al. 2018, A\&A, 618, A93

Cardelli, J. A., Clayton, G. C., \& Mathis, J. S. 1989, ApJ, 345, 245

Casagrande, L., Ramírez, I., Meléndez, J., Bessell, M., \& Asplund, M. 2010, A\&A, 512, A54

Chabrier, G., Gallardo, J., \& Baraffe, I. 2007, A\&A, 472, L17
Chen, Y., Girardi, L., Bressan, A., et al. 2014, MNRAS, 444, 2525

Choi, J., Dotter, A., Conroy, C., et al. 2016, ApJ, 823, 102

Claret, A., \& Bloemen, S. 2011, A\&A, 529, A75

Clausen, J. V., Bruntt, H., Claret, A., et al. 2009, A\&A, 502, 253

Curtis, J. L. 2016, PhD thesis, Penn State Univ.

Curtis, J. L., Wolfgang, A., Wright, J. T., Brewer, J. M., \& Johnson, J. A. 2013, AJ, 145,134

Etzel, P. B. 1981, in Proc. NATO Adv. Study Inst., Photometric and Spectroscopic Binary Systems, ed. E. B. Carling \& Z. Kopal (Dordrecht: Reidel), 111

Feiden, G. A., \& Chaboyer, B. 2013, ApJ, 779, 183

Feiden, G. A., \& Chaboyer, B. 2014, ApJ, 789, 53

Flower, P. J. 1996, ApJ, 469, 355

Foreman-Mackey, D. 2016, JOSS, 1, 24

Foreman-Mackey, D., Hogg, D. W., Lang, D., \& Goodman, J. 2013, PASP, 125,306

Fűrész, G. 2008, PhD thesis, Univ. Szeged

Furlan, E., Ciardi, D. R., Everett, M. E., et al. 2017, AJ, 153, 71

Gaia Collaboration, Brown, A. G. A., Vallenari, A., et al. 2018, A\&A, 616, A1 Gelman, A., \& Rubin, D. B. 1992, StaSc, 7, 457

Gilliland, R. L., Jenkins, J. M., Borucki, W. J., et al. 2010, ApJL, 713, L160 Goodman, J., \& Weare, J. 2010, CAMCS, 5, 65

Gregory, P. C. 2005, ApJ, 631, 1198

Hartman, J. D., Quinn, S. N., Bakos, G. Á., et al. 2018, AJ, 155, 114

Hełminiak, K. G., Konacki, M., Maehara, H., et al. 2019, MNRAS, 484, 451

Henden, A. A., Levine, S., Terrell, D., \& Welch, D. L. 2015, AAS Meeting, 225, 336.16

Hilditch, R. W. 2001, An Introduction to Close Binary Stars (Cambridge: Cambridge Univ. Press)

Høg, E., Fabricius, C., Makarov, V. V., et al. 2000, A\&A, 355, L27

Hora, J. L., Luppini, G. A., \& Hodapp, K.-W. 1994, Proc. SPIE, 2198, 498

Huang, Y., Liu, X.-W., Yuan, H.-B., et al. 2015, MNRAS, 454, 2863

Husser, T.-O., Wende-von Berg, S., Dreizler, S., et al. 2013, A\&A, 553, A6 Ireland, M. J. 2013, MNRAS, 433, 1718

Irwin, J. M., Quinn, S. N., Berta, Z. K., et al. 2011, ApJ, 742, 123

Kipping, D. M. 2010, MNRAS, 408, 1758

Kraus, A. L., Cody, A. M., Covey, K. R., et al. 2015, ApJ, 807, 3

Kraus, A. L., Ireland, M. J., Huber, D., Mann, A. W., \& Dupuy, T. J. 2016, AJ, 152,8

Kraus, A. L., Ireland, M. J., Martinache, F., \& Lloyd, J. P. 2016, ApJ, 679, 762 Latham, D. W., Stefanik, R. P., Torres, G., et al. 2002, AJ, 124, 1144

Lindegren, L., Hernández, J., Bombrun, A., et al. 2018a, A\&A, 616, A2

Lindegren, L., Hernández, J., Bombrun, A., et al. 2018b, Gaia DR2 Astrometry Presentation, 35, https://www.cosmos.esa.int/documents/29201/1770596/ Lindegren_GaiaDR2_Astrometry_extended.pdf/1ebddb25-f010-6437cb14-0e360e2d9f09

Mandel, K., \& Agol, E. 2002, ApJL, 580, L171

Morales, J. C., Gallardo, J., Ribas, I., et al. 2010, ApJ, 718, 502

Mullan, D. J., \& MacDonald, J. 2001, ApJ, 559, 353

Nordström, B., Latham, D. W., Morse, J. A., et al. 1994, A\&A, 287, 338

Olivares, J., Bouy, H., Sarro, L. M., et al. 2019, A\&A, 625, A115

Popper, D. M., \& Etzel, P. B. 1981, AJ, 86, 102

Prša, A., Harmanec, P., Torres, G., et al. 2016, AJ, 152, 41

Skrutskie, M. F., Cutri, R. M., Stiening, R., et al. 2006, AJ, 131, 1163

Somers, G., \& Pinsonneault, M. H. 2015, MNRAS, 449, 4131

Szentgyorgyi, A. H., \& Fúrész, G. 2007, RMxAC, 28, 129

Torres, G. 2010, AJ, 140, 1158

Torres, G. 2013, AN, 334, 4

Torres, G., Andersen, J., \& Giménez, A. 2010, A\&ARv, 18, 67

Torres, G., Curtis, J. L., Vanderburg, A., Kraus, A. L., \& Rizzuto, A. 2018, ApJ, 866, 67

Torres, G., Lacy, C. H., Marschall, L. A., et al. 2006, ApJ, 640, 1018

Torres, G., Neuhäuser, R., \& Guenther, E. W. 2002, AJ, 123, 1701

Torres, G., Vaz, L. P. R., \& Sandberg Lacy, C. H. 2008, AJ, 136, 2158

Vanderburg, A., \& Johnson, J. A. 2014, PASP, 126, 948

Vanderburg, A., Latham, D. W., Buchhave, L. A., et al. 2016, ApJS, 222, 14

Zacharias, N., Finch, C. T., Girard, T. M., et al. 2013, AJ, 145, 44

Zucker, S., \& Mazeh, T. 1994, ApJ, 420, 806 